\title{
Edge Enhancement by Diffusion in Microscopic Magnetic Resonance Imaging
}

\author{
B. Pütz, D. Barsky, and K. Schulten*
}

Beckman Institute and Dept. of Physics, University of Illinois

405 N. Mathews Avenue, Urbana, Illinois 61801

July 23, 1991

*To whom correspondences should be sent. 


\section{Abstract}

In microscopic MRI ( $\sim 10 \mu$ m resolution), diffusion boundaries impermeable to water on a millisecond time scale distort the line shape function of the observed frequency spectra from the transverse magnetization in a manner similar to motional narrowing in MR spectroscopy. Reconstruction techniques developed for macroscopic imaging interpret these distortions as spatial deformations of objects. This distortion of frequency spectra is demonstrated for geometrically simple objects, and it is shown how in such cases the reconstructed images should be interpreted and how diffusioninduced distortions in frequency space may actually be exploited to enhance image contrast around compartmental boundaries. The distortions are properly described by Kubo's line shape function for which suitable numerical algorithms are provided. A parameter is introduced that provides an estimation of the extent of diffusioninduced distortions which can vary from an enhancement of image intensity near boundaries to, for extreme motional narrowing, a focusing of image intensity around compartment centers. 


\section{Introduction}

The investigation of microscopic objects, such as single cells, by the method of magnetic resonance imaging (MRI), requires an understanding of the effects of diffusion in order to correctly interpret the obtained images (5). On the time scale (milliseconds) of typical measurements, water molecules can diffuse a distance as large or larger than the spatial resolution of the instrument (1) which is presently below $10 \mu \mathrm{m}$ and may become even finer $(2,3)$. As a result, techniques of image reconstruction from frequency profile data that work well for conventional, macroscopic MRI, can lead to distorted density profiles of images in MRI microscopy. As we will show, these distortions actually add new image contrasts, and a proper interpretation of these can be utilized to the advantage of microscopic MRI.

The physical origin of the distortions is the diffusion of water molecules near impermeable compartmental walls such as membranes. This diffusion affects the frequency-position relation of MRI spectra. The actual relation must be described by the theory of line shape functions (4). In this article we determine the effect of diffusion on the line shape function for several geometric settings which are prototypical for microscopic biological tissues. We first investigate a one-dimensional diffusion space to develop a theoretical understanding of the diffusion-induced effects. We then consider spins diffusing in a sphere, spins diffusing in a spherical shell, and, finally, spins diffusing in a sphere as well as in a disjunct shell surrounding the sphere. The 
spheres may be viewed as structurally idealized models of biological compartments like liposomes, air bubbles, or single cells. For the sake of simplicity we assume all physical boundaries to be impermeable on the time scale of an MRI measurement.

Towards the end of the paper, a brief discussion of experimental realities is given which addresses the consequences of permeable compartmental walls, natural line widths, and other practical limitations. Some of these considerations are further discussed in Chapter 4 of Ref. (5).

For a confirmation of an approach based on the theory of line shape functions we describe computer simulations of diffusion-mediated relaxation processes in the same geometrical settings. The simulations yield results close to those obtained by calculations involving the theory of line shape functions. Both calculation schemes predict that, interestingly, diffusion effects do not blur an image as one may expect at first, but rather result in a contrast enhancement of diffusion boundaries, in halos, and in point-like artifacts. These effects will be presented in images that have been reconstructed from simulated data.

The work presented here is an extension of results compiled in (6). 


\section{Theory}

At the most elementary level the MRI procedure requires one to extract spatial information from frequency profiles. The process of reconstruction begins with spin density distributions derived from frequency spectra. This derivation requires a precise knowledge of the frequency-position relation, usually provided by a magnetic field

$$
\vec{H}=\left(H_{o}+G z\right) \hat{z}
$$

which results from the superposition of a linear gradient $G z$ with a homogeneous field $H_{o}$. Ordinarily, because every spin's precession frequency is proportional to the local magnetic field, the frequency spectrum $I(\omega)$ faithfully mirrors the spin density $\rho(z)$, that is, $I(\omega) \propto \rho\left(\omega_{o}+\gamma G z\right)$ where $\gamma$ denotes the gyromagnetic ratio of protons and $\omega_{o}=\gamma H_{o}$. This familiar relationship is illustrated in Fig. 1. Unfortunately, in microscopic MRI, diffusion can render the simple frequency-position relation invalid, even when [1] remains correct.

Diffusion is generally expected to contribute to blurring of images. For example, studies on paramagnetic contrast agents have shown that biological contours are blurred since contrast agents affect not only the tissue compartments to which they are confined but, through diffusive water exchange, also affect surrounding regions $(7,8,9,10,11)$. Thus, one might expect that diffusion blurring should become 
prevalent in microscopic imaging, yet this may not necessarily be the case.

When one examines the effects of diffusion within microscopic compartments, one predicts a distortion of the frequency spectrum of the transverse magnetization due to the well-known effect of motional narrowing (12). Figure 1 suggests how motional narrowing can falsely indicate a sharply peaked density of spins where in fact the spins uniformly populate the sampling region. If one assumed the usual relation, $\rho(z) \propto I\left(z_{o}+\omega / \gamma G\right)$ where $z_{o}=\omega_{o} / \gamma G$, in interpreting a motion-narrowed spectrum such as that shown dashed in Fig. 1, then after image reconstruction one would arrive at an artificially narrow density distribution. This implies that compartments may appear smaller than they actually are. As we will demonstrate below, other artifacts can appear which do not directly reflect the actual spin density distribution.

We now derive the line shape function, which describes the frequency response of the transverse magnetization of water protons (spins). The line shape function is obtained by a Fourier transform of the observed FID signal. A theoretical description of this signal can be based on the diffusion-Bloch equation (13)

$$
\partial_{t} m_{\perp}=\left(\nabla \cdot D \nabla-i \Omega(\vec{r})-\frac{1}{T_{2}}\right) m_{\perp} .
$$

This equation is obeyed by the transverse magnetization $m_{\perp}=m_{x}+i m_{y}$ of a spin ensemble diffusing in an external magnetic field. The diffusion constant $D$ characterizes the rate of diffusion; the angular precession frequency $\Omega(\vec{r})$ is proportional to the strength of the local field $H(\vec{r}) \hat{z}$, i. e., $\Omega(\vec{r})=\gamma H(\vec{r})$; and $T_{2}$ is the native relaxation 
time.

An analytical solution of [2] can be obtained for spins diffusing in an unbounded space in the presence of the magnetic field [1]. To derive this solution we first separate out the native relaxation and the precession due to the homogeneous field $H_{o}$ by defining $m_{\perp}=m_{\perp}^{\prime} \exp \left(-i \omega_{L}-1 / T_{2}\right)$, where $\omega_{L}=\gamma H_{o}$. This yields instead of [2]

$$
\partial_{t} m_{\perp}^{\prime}=\left(D \partial_{z}^{2} \pm i \gamma G z\right) m_{\perp}^{\prime}
$$

A solution for an initial Gaussian distribution of magnetization

$$
m^{\prime}(z, t=0)=\frac{1}{\sqrt{\pi \delta^{2}}} \exp \left(-\frac{(z-\bar{z})^{2}}{\delta^{2}}\right)
$$

which satisfies the boundary conditions

$$
m^{\prime}(z= \pm \infty, t)=0
$$

is

$$
m^{\prime}(z, t)=\frac{1}{\sqrt{\pi \delta^{2}\left(1+\frac{4 D t}{\delta^{2}}\right)}} \exp \left[-\frac{\left((z-\bar{z}) \pm i D \gamma G t^{2}\right)^{2}}{\delta^{2}\left(1+\frac{4 D t}{\delta^{2}}\right)} \pm i \gamma G z t-\frac{\left(D \gamma^{2} G^{2} t^{3}\right)}{3}\right]
$$

A derivation is provided in Appendix A. This result generalizes the well-known formula of Hahn and Torrey $(14,13)$ and is very useful to test numerical algorithms, such as the simulation program presented below. We reiterate that [6] describes the case of unbounded diffusion.

We next derive an expression of the line shape function for the case of diffusion in a bounded spatial domain. Mathematically, the boundaries of the domain impose 
boundary conditions on the solution of [2], namely, that the diffusive flux (or, equivalently, the magnetic flux) vanishes at the boundary. For a one-dimensional domain with boundaries at $\pm z_{b}$, one has the conditions

$$
\left.\partial_{z} m_{\perp}(z, t)\right|_{z= \pm z_{b}}=0
$$

The other diffusion domains considered below are three-dimensional with spherical boundaries, and for those the boundary conditions corresponding to [7] require that the radial component of the gradient of $m_{\perp}(\vec{r}, t)$ vanishes at the barrier.

The solution of [4] requires one to specify an initial condition. In both one and three dimensions, we assume an initial condition of a uniform, normalized transverse magnetization

$$
m_{\perp}(z, t=0)=m_{\perp}(\vec{r}, t=0)=1 .
$$

as occurs experimentally after a $90^{\circ}$ pulse.

For the moment we consider only one-dimensional diffusion. We proceed to solve diffusion-Bloch equation [2] by noticing that it has the formal solution ${ }^{1}$

$$
m_{\perp}(z, t)=e^{\left(\hat{D}+i \Omega(z)-1 / T_{2}\right) t} m_{\perp}(z, 0)
$$

where $\hat{D}=D \partial_{z}^{2}$ is the diffusion operator for a position-independent diffusion coefficient $D$ and $\Omega(z)=\gamma\left(H_{o}+G z\right)$.

\footnotetext{
${ }^{1}$ The diffusion constant will be written as $D$, and the diffusion operator $\nabla \cdot D \nabla$ written as $\hat{D}$.
} 
The total magnetization is given by a spatial average over the local magnetization

$$
\begin{aligned}
M(t)=\int d z m_{\perp}(z, t) & =\int d z e^{\left(\hat{D}+i \Omega(z)-1 / T_{2}\right) t} m_{\perp}(z, 0) \\
& =\left\langle 1 e^{\left(\hat{D}+i \Omega(z)-1 / T_{2}\right) t} p_{o}\right\rangle .
\end{aligned}
$$

The brackets $\langle\ldots\rangle$ denote the integral. The magnetization $m_{\perp}(z, 0)$ is initially equal to the (normalized) equilibrium distribution $p_{o}(z)$.

The real part of the Fourier transform of $M(t)$ gives the frequency spectrum

$$
\begin{aligned}
I(\omega) & =\frac{1}{2 \pi} \operatorname{Re} \int_{-\infty}^{+\infty} d t e^{i \omega t} M(t) \\
& =\frac{1}{2 \pi} \operatorname{Re}\left\langle 1 \frac{1}{\hat{D}+i(\Omega(z)-\omega)-1 / T_{2}} p_{o}\right\rangle .
\end{aligned}
$$

This expression, except for the relaxation term $1 / T_{2}$, was first derived by Kubo $(4,15) . I(\omega)$ is the line shape function for a spin ensemble diffusing in a linear gradient field.

Expression [10] cannot be evaluated analytically. A numerical solution can be obtained by invoking a finite difference approximation. To accomplish this, the diffusion space is divided into $n$ equal intervals of length $\Delta z=2 z_{b} / n$ along the $z$-axis. For $k=1, \ldots, n$ the $k^{\text {th }}$ interval, denoted by $[k]$, is centered at $z_{k}=-z_{b}+(2 k-1) \Delta z$ such that $z_{1}=-z_{b}+\frac{z_{b}}{n}$ and $z_{n}=+z_{b}-\frac{z_{b}}{n}$. The finite difference approximation represents functions $f(z)$ by $n$-dimensional vectors. The equilibrium distribution $p_{o}$, for example, becomes a vector $\vec{p} \in \mathbb{R}^{n}$ with $k^{\text {th }}$ component

$$
p_{k}=p_{o}\left(z_{k}\right), \quad k=1, \ldots, n
$$


i. e., $p_{k}$ is proportional to the spin population in the interval $[k]$.

To evaluate [10] we define an operator $\hat{O}(\omega)=\hat{D}+i[\Omega(z)-\omega]-1 / T_{2}$ for each frequency value $\omega$ at which we want to know the intensity. The calculation of the inverse of $\hat{O}(\omega)$ - as required in [10] — by means of a finite difference scheme is not straightforward. In order to evaluate [10], we notice that in a discretized form the right hand side of [10] can be written $\frac{1}{2 \pi} \operatorname{Re}\left\langle\overrightarrow{1} \mathcal{O}^{-1}(\omega) \vec{p}\right\rangle$, where the brackets now represent a summation over $k$ instead of an integral and $\mathcal{O}$ represents the discrete matrix form of $\hat{O}$. Rather than evaluating $\vec{c}(\omega)=\mathcal{O}^{-1}(\omega) \vec{p}$ through matrix inversion of $\mathcal{O}(\omega)$, we solve the linear equation

$$
\mathcal{O}(\omega) \vec{c}(\omega)=\vec{p}
$$

for the unknown vector $\vec{c}(\omega)$. To accomplish this we have employed a Gauss-Seidel elimination scheme (16). This method of evaluating [10] was suggested and applied in $(17)$ for line shape functions of electron spins. ${ }^{2}$

The algorithm suggested requires one to discretize $\hat{O}(\omega)$ into a complex $n \times n$ matrix $\mathcal{O}(\omega)$. We will first consider the discretization for the case of one-dimensional diffusion, and later generalize the method to three-dimensional diffusion. After dis-

\footnotetext{
${ }^{2}$ The present work was undertaken after one of the authors (K. S.) remarked to Paul Lauterbur in 1989 that the line shape function function shown in Fig. 1 of this reference qualitatively describes the effect of diffusion between two parallel plates on a frequency distribution of a spin ensemble and should also describe diffusion effects on images reconstructed in MRI.
} 
cretization, $\Omega(z)$ and $1 / T_{2}$ contribute a term $i\left(\Omega_{k}-\omega\right)-1 / T_{2}$ to the diagonal of $\mathcal{O}(\omega)$, where in this notation, $\Omega_{k}=\Omega\left(z_{k}\right)$.

The diffusion operator $\hat{D}$ describes the exchange of particles between intervals (elements of $\vec{p}$ ) and will contribute to off-diagonal elements of $\mathcal{O}(\omega)$. To discretize $\hat{D}$ we choose the representation suggested in $(18,19,20,21)$ which satisfies detailed balance, a desirable property for the discretization of $\hat{D}$ in dimensions higher than one. For one-dimensional diffusion, $\hat{D}$ can be represented by a tridiagonal matrix $(18,20,21,22)$

$$
\hat{D}(z) \rightarrow \mathcal{D}_{j k}=\left\{\begin{array}{cl}
-\left(\mathcal{D}_{k-1, k}+\mathcal{D}_{k+1, k}\right) & j=k \\
\tau^{-1} \sqrt{\frac{p_{j}}{p_{k}}} & j=k \pm 1 \\
0 & \text { otherwise }
\end{array}\right.
$$

where $\tau^{-1} \sqrt{\frac{p_{j}}{p_{k}}}$ denotes the jump rate from interval $[k]$ to the neighboring interval $[j]$ and is related to the diffusion constant by $\tau^{-1}=D /(\Delta z)^{2}$. This last formula is the correct finite difference analogue to Einstein's diffusion relation $(17,23)$. In this representation $\mathcal{D}_{j k}$ denotes the diffusion rate from interval $[k]$ to $[j]$ and $\mathcal{D}_{j j}$ controls the flow from interval $[j]$ to intervals $[j-1],[j+1]$. The elements $\mathcal{D}_{-1,1}$ and $\mathcal{D}_{n+1, n}$ appearing in [13] are set to zero in accordance with the boundary conditions (18). 
The matrix $\mathcal{O}(\omega)$ that provides the discrete representation of $\hat{O}$ is then

$$
\mathcal{O}_{j k}=\left\{\begin{array}{cl}
-\left(\mathcal{O}_{k-1, k}+\mathcal{O}_{k+1, k}\right)+i\left(\Omega_{k}-\omega\right)-1 / T_{2} & j=k \\
\tau^{-1} \sqrt{\frac{p_{j}}{p_{k}}} & j=k \pm 1 \\
0 & \text { otherwise }
\end{array}\right.
$$

The calculation described is carried out for a series of $\omega$ values in the expected range of the frequency distribution. For each choice of $\omega$ the unknown $\vec{c}(\omega)$ defined in [12] is determined and $I(\omega)$ evaluated according to

$$
I(\omega)=\frac{1}{2 \pi} \operatorname{Re}\left\langle\overrightarrow{1} \hat{O}^{-1}(\omega) \vec{p}\right\rangle=\frac{1}{2 \pi} \operatorname{Re}(\overrightarrow{1} \cdot \vec{c}(\omega))=\frac{1}{2 \pi} \sum_{k=1}^{n} \operatorname{Re}\left(c_{k}(\omega)\right) .
$$

In order to illustrate this expression, we present in Fig. 2 the contributions $I\left(\omega, z_{k}\right)=\operatorname{Re}\left(c_{k}(\omega)\right)$ to [15]. The quantity $I\left(\omega, z_{k}\right)$ can be interpreted as the frequency distribution of spins which are initially placed at position $z=z_{k}$ and are then free to diffuse. For fixed spins this quantity is $I(\omega, z) \propto \delta(\omega-\gamma G z)$; i. e., in this case $I(\omega, z)$ is centered narrowly along the diagonal of the $(\omega, z)$-plane. Fig. 2 shows that diffusion distorts $I(\omega, z)$. The maximum of $I(\omega, z)$ mostly follows the diagonal of the $(\omega, z)$-plane, however, diffusion results in a broadening of the $\omega$-dependence for any fixed $z$. Near the boundaries $z=-z_{b}$ and $z=+z_{b}$ of the diffusion space, the frequencies of fixed spins are at $\gamma\left(H_{o}-G z_{b}\right)$ and $\gamma\left(H_{o}+G z_{b}\right)$, respectively, i. e., at the boundaries of the $\omega$-interval shown in Fig. 2. Diffusion shifts the maxima of $I\left(\omega, z_{k}\right)$ towards the center of the $\omega$-interval. These shifts result in a narrowing of the total frequency distribution $I(\omega)[15]$ as discussed further below. 
We now generalize the above algorithm to three-dimensional diffusion domains. A straightforward application of the algorithm would lead one to a three-dimensional discretization scheme for $\hat{O}(\omega)$ such as considered in (20) which yields matrices $O_{j k}$ of dimensions $n \approx 10^{6}$. Solving [12] for such large dimensions is very formidable even though the matrix $O_{j k}$ is very sparse (21, 24). To avoid such time-consuming numerical procedures, we have adapted an approximation scheme that yields very satisfactory results, as will be demonstrated below by comparison with simulations.

An approximate expression for the line shape function $I(\omega)$ for higher dimensional diffusion can be developed by modifying the equilibrium population vector $\vec{p}$ to represent the appropriate density profile along the $z$-axis, i. e., along the direction of the field gradient. The components $p_{k}$ take the form

$$
p_{k} \propto\left\{\begin{array}{cl}
1 & \text { linear geometry } \\
r^{2}-z_{k}^{2} & \text { spherical geometry }
\end{array}\right.
$$

where $r$ is the radius of the object and $-r \leq z_{k} \leq r, z_{k}=-r+(2 k-1) r / n$ for $k=1, \ldots, n$. We normalize $p_{k}$ such that $\sum_{k} p_{k}=1$. The approximation involved in this description assumes that all spins with the same $z$ value yield identical contributions to the spectrum $I(\omega)$. This, however, is not necessarily the case since the compartmental boundaries will usually depend on the $x, y$-coordinates, and, hence, spins with identical $z$-coordinate but different $x, y$-coordinates are not equivalent with respect to diffusion. The approximation involved here replaces an $x, y$-dependence of 
the line shape function by an average value which depends only on $z_{k}$. Comparisons with complete simulations (presented below) reveal that the suggested approximation is quite accurate.

The theory described applies only to simple, undivided diffusion spaces. Cases of practical interest, such as a sphere surrounded by solvent, can be dealt with by dividing diffusion spaces and evaluating line shape functions separately for each compartment. For example, a spherical compartment of radius $r_{i}$ surrounded by solvent can be described in three steps:

(i) Obtain the frequency spectrum $I_{(i)}(\omega)$ of a sphere of radius $r_{i}$.

(ii) Obtain the frequency spectrum $I_{(i i)}(\omega)$ inside the spherical shell, limited by an inner radius $r_{i}$ and an outer radius $r_{a}$ using

$$
p_{k} \propto\left\{\begin{array}{cc}
r_{a}^{2}-z_{k}^{2} & r_{i}<\left|z_{k}\right|<r_{a} \\
r_{a}^{2}-r_{i}^{2} & 0<\left|z_{k}\right|<r_{i}
\end{array}\right.
$$

where $z_{k}=-r_{a}+(2 k-1) r_{a} / n, k=1,2, \ldots, n$. Again, this is only an approximation in the sense that the $x, y$-dependence of the line shape function has been replaced by an average.

(iii) Scale and combine the frequency spectra (i) and (ii)

$$
I(\omega) \propto S_{(i i)} V_{(i)} I_{(i)}(\omega)+S_{(i)} V_{(i i)} I_{(i i)}(\omega)
$$


where $S_{(\alpha)}=\int d \omega I_{(\alpha)}(\omega), \alpha=i$, ii denotes the integral over the signal intensity in each subvolume $V_{(i)}=\frac{4 \pi}{3} r_{i}^{3}$ and $V_{(i i)}=\frac{4 \pi}{3}\left(r_{a}^{3}-r_{i}^{3}\right)$. [18] implies the de-

sired property, that the ratio of the integrals $\tilde{S}_{(i)}(\omega)=\int d \omega S_{(i i)} V_{(i)} I_{(i)}(\omega)$ and $\tilde{S}_{(i i)}(\omega)=\int d \omega S_{(i)} V_{(i i)} I_{(i i)}(\omega)$ is equal to the ratio of the corresponding volumes, i. e.,

$$
\frac{V_{(i)}}{V_{(i i)}}=\frac{\tilde{S}_{(i)}}{\tilde{S}_{(i i)}} .
$$

\section{A Simulation Program}

We have developed a simulation algorithm to investigate relaxation and diffusion effects in one, two, and three dimensions. This algorithm does not require the approximations mentioned above. Since the simulations are very computer time-consuming, calculations have been carried out only to demonstrate the accuracy of the line shape function description, in particular, for the case of three-dimensional geometries, for which the line shape function description suggested above is only approximate.

For a particular native relaxation rate and external magnetic field strength, the program simulates independent "spins" (spin ensembles) by solving the Bloch equations classically and representing diffusion as random walk. The particles are assigned diffusion steps according to

$$
\vec{r}\left(t_{n+1}\right)=\vec{r}\left(t_{n}\right)+\sqrt{2 D \Delta t} \vec{\xi}
$$


where the components $\xi_{i}$ of the random vector $\vec{\xi}$ are uncorrelated random numbers obeying the distribution

$$
p\left(\xi_{i}\right) d \xi_{i}=\frac{1}{\sqrt{2 \pi}} e^{-\xi_{i}^{2} / 2} d \xi_{i} .
$$

The time steps $\Delta t=t_{n+1}-t_{n}$ are chosen short enough to ensure that the time evolution of the system is represented appropriately.

The relaxation of the magnetization is separated into a longitudinal component $m_{z}$ and into a transverse component $m_{\perp}=m_{x}+i m_{y}$. An explicit finite difference approximation to the Bloch equation can then be written

$$
\begin{array}{lll}
m_{z}\left(t_{n+1}\right) & =\left(m_{z}\left(t_{n}\right)-m_{z}^{o}\right) e^{-\Delta t / T_{1}}+m_{z}^{o} \\
m_{\perp}\left(t_{n+1}\right) & = & m_{\perp}\left(t_{n}\right) e^{-\Delta t / T_{2}-i \bar{\omega} \Delta t}
\end{array}
$$

where $m_{z}^{o}$ is the equilibrium magnetization in the local magnetic field $\vec{B}\left(\vec{r}\left(t_{n+1}\right)\right)$, and where $\bar{\omega}$ is the mean Larmor frequency over one time step $\Delta t$. A more detailed description of the simulation program is provided in Appendix B.

We are primarily interested in the time evolution of the transverse magnetization of the whole spin ensemble, $M_{\perp}(t)=\sum_{\text {all spins }} m_{\perp}(t) . M_{\perp}(t)$ is acquired by having the program write $M_{\perp}\left(t_{n}\right)$ to a file at regular time intervals, e. g., $10 \Delta t$. This data file can be thought of as recorded from an actual experiment. The frequency spectrum for the simulated spin ensemble is obtained by taking the Fourier transform of $M_{\perp}(t)$. Since $m_{\perp}(t)$ is available only at the discrete times $t_{n}$, it follows that $M_{\perp}(t)$ will be known at only these times, too. A fast Fourier transform (FFT) of the (discrete) 
data set, $\left\{M_{\perp}\left(t_{n}\right)\right\}$, will yield the discrete, complex frequency spectrum $\tilde{M}\left(\omega_{k}\right)$. Any reconstruction procedure must utilize the absorption signal which corresponds to the real part of $\tilde{M}\left(\omega_{k}\right)(25,26)$, denoted here by $I\left(\omega_{k}\right)$. In symbolic form, the procedure is

$$
I\left(\omega_{k}\right)=\operatorname{Re}\left(\mathrm{FFT}\left[\left\{M_{\perp}\left(t_{n}\right)\right\}\right]\right) .
$$

The simulation results have been compared in (6) (not shown here) for onedimensional diffusion with the analytical solution [6] to calibrate the accuracy of the simulation, e.g., the necessary sample size. In Fig. 3 below we compare results of simulations of one-dimensional diffusion with the description based on the line shape function [10]. The simulation program allows one to describe spin frequency distributions also for three-dimensional diffusion, in principle to any desired accuracy, and, thereby, serves as a standard to which one can compare the approximate description suggested above. 


\section{Numerical Results}

To demonstrate the effects of motional narrowing on frequency spectra and to understand the impact of these effects on reconstructed images, we consider various simple geometrical settings. In each case we show frequency spectra calculated according to the line shape function formalism [15] as well as by simulation [23] for different diffusion constants. This will reveal the influence of diffusion on the line shape function, and will also provide a comparison of the theoretical methods.

The first setting consists of a linear diffusion space with impermeable boundaries. This can be realized by a cube filled with water and a gradient parallel to one of the edges: all the spins within a plane perpendicular to the gradient cannot be discerned, and the problem can be reduced to one dimension. The following parameters describe the one-dimensional diffusion space as well as the three-dimensional objects to be presented later: a length (or diameter) of $40 \mu \mathrm{m}$, four diffusion constants in the range $3 \cdot 10^{-4} \ldots 3 \cdot 10^{-7}$, and a field gradient of $0.02 \mathrm{~T} / \mathrm{m}$.

Figure 3 presents frequency spectra for a one-dimensional diffusion interval for four different diffusion constants, the values of which are representative of those occurring in biological tissues. Spectrum (c) corresponds to diffusion in pure water. The two extreme cases of fast and slow diffusion show what was suggested above. For slow diffusion (a) the spectrum closely reflects the uniform spin density; it is flat 
(undistorted) across the center and narrowly peaked very close to the boundaries. For fast diffusion (d) the spectrum exhibits a singly peaked shape typical of motional narrowing.

In all cases presented in Fig. 3, the spectra resulting from the theory of line shape functions and those obtained through simulations agree well. The fluctuations in the simulated spectra are due to finite samples as can be seen by the following argument. The total sample of 160000 particles simulated corresponds to an average number of about 1250 particles in each of the 128 discretization bins which represented the continuous frequency domain. As a result, fluctuations of the order of $3 \%(\sim 1 / \sqrt{N})$ are expected. Nevertheless, Fig.3 shows that the simulation closely reproduces the line shape function, and one can conclude that in the case of one-dimensional diffusion, the simulation program yields accurate results. The simulation can then be used in turn to check approximations introduced to describe line shape functions for threedimensional diffusion domains.

The spectra in Fig. 3 (b and c) reveal how as the diffusion constant increases, the two peaks located at the boundaries in Fig. 3 (a) move toward the center. With increasing diffusion constants the two peaks merge and, in limit of high motional narrowing, produce the single peak observed in Fig. 3 (d).

The intensity maxima seen in Fig. 3 can be understood as follows. Diffusing spins do not precess at a constant Larmor frequency, but rather experience the local 
frequencies along their diffusion paths. The spectra observed reflect the diffusive movements of the spins. The observed magnetization signal conveys only the average frequency over one full precession of the magnetization vector ${ }^{3}$ (4). In Fig. 4 various diffusion trajectories are visualized. Schematic (straight) diffusion trajectories have been drawn for time intervals $\Delta T \approx 2 \pi / \gamma G \Delta z$ that are comparable to a period of precession. In case of slow diffusion (a) the mean positions of the diffusion trajectories are distributed evenly over the whole diffusion interval. In the case of fast diffusion (c) 'reflection' at the boundaries pushes the mean positions towards the center of the diffusion interval. As a result, the frequency of the resonance signal-actually the average frequency over a time interval of length $\Delta T$-will be shifted towards the center frequency.

The spectra presented in Fig. 3 imply that the particle density $\rho(z)$ will be misrepresented if it is assumed to be proportional to the signal intensity, i. e., $\rho(z) \propto I(\Omega(z))$. Image reconstruction based on this assumption will transform diffusion-induced distortions of frequency spectra into diffusion-induced distortions of spin density; i. e., distortions will appear as low density (dark region) at the boundaries and high density (bright region) at positions corresponding to the peaks of $I(\omega)$. Interpreting Fig. 3 (d)

\footnotetext{
${ }^{3}$ Since only differences in the precession frequency are relevant in determining the location of the signal origins, it is only the differential precession due to the gradient field, and not the precession due to the homogeneous component of the magnetic field, that has to be considered.
} 
without these considerations would lead to the erroneous assignment of a large spin density at the center of the diffusion space.

It is desirable to derive a control parameter which allows one to estimate to what extent bounded diffusion will distort the frequency distribution and, thereby, the reconstructed image. Such a parameter should depend on the gradient $G$, the size of the diffusion interval in the direction of the gradient, and the diffusion constant $D$.

The following considerations attempt to identify the time scale and the spatial scale over which diffusion induces a nuclear spin to experience dephasing relative to precession at its average position. We provide two avenues to obtain such parameters.

A first avenue identifies the parameters through inspection of solution [6] for the magnetization of diffusing spins in a field with a linear gradient $G$. The last term in the exponential of [6] describes a position-independent relaxation which can be written as a factor $\exp \left[-\left(t / \tau_{1}\right)^{3}\right]$ where the time scale $\tau_{1}$ of relaxation is given by

$$
\tau_{1}^{-1}=\sqrt[3]{\frac{D \gamma^{2} G^{2}}{3}} .
$$

This result, briefly discussed in Appendix A (see [35]), was given by Hahn and Torrey $(14,13)$. One can also obtain a spatial scale $\delta_{1}$ by considering the second term in the exponent of $[6]$ and writing its contribution as a factor $\exp \left[ \pm i \frac{z}{\delta_{1}} \frac{t}{\tau_{1}}\right]$ where the length scale $\delta_{1}$ is

$$
\delta_{1}=\sqrt[3]{\frac{D}{3 \gamma G}} .
$$


A second avenue to obtain parameters which allow one to estimate the effect of diffusion on MRI images involves the following qualitative considerations. Diffusion implies that over a time scale $\tau_{2}$, position differences develop on a length scale $\delta_{2}$ determined by

$$
\delta_{2}^{2} \sim D \tau_{2}
$$

At the same time, the existence of a field gradient $G$ implies that two spins at locations that are separated by a distance $\delta_{2}$ along the gradient experience dephasing on the time scale $\tau_{2}$ determined by

$$
\tau_{2}^{-1} \sim \frac{\gamma G \delta_{2}}{\pi}
$$

One can now solve the two relationships [26], [27] for either $\delta_{2}$ or $\tau_{2}$. Using [26] and eliminating $\delta_{2}$ in [27] yields

$$
\tau_{2}^{-1} \sim \sqrt[3]{\frac{D \gamma^{2} G^{2}}{\pi^{2}}}
$$

an expression which reproduces the Hahn and Torrey $(14,13)$ result $[24]$ except for a factor of about 0.6. Using [26] to eliminate instead $\tau_{2}$ yields $^{4}$

$$
\delta_{2} \sim \sqrt[3]{\frac{\pi D}{\gamma G}}
$$

which reproduces [25] except for a factor of about 2 .

\footnotetext{
${ }^{4}$ For heuristic reasons, we have kept an extra factor of $\pi$ in expression [29] while disregarding all other numerical factors: the resulting parameter $q$ assumes the numerical value $q=1$ for values of $G$ and $D$ at which edge intensity peaks just begin to merge to a single central intensity peak.
} 
To interpret the resulting length scale of [29], we note that both [26] and [27] establish a relation between time and length - a relation which, in [26] stems from diffusion laws, and in [27] is a consequence of dephasing by a field gradient. In deriving [29] we set the two time scales equal, implying that $\delta_{2}$ is the length of the diffusion path over which a particle gets out of phase by $2 \pi$ compared to a spin with fixed position. In Fig. $4 \delta_{2}$ as given by [29] would correspond to the net displacement of the paths shown if we set $\Delta T=\tau_{2}$.

Expression [29] provides an estimate of the distance from the boundary at which the intensity peaks in Fig. 3 appear. According to [29] the following $\delta_{2}$-values correspond to Fig. 3 (a, b, c, d): $2.3 \mu \mathrm{m}, 4.8 \mu \mathrm{m}, 10 \mu \mathrm{m}, 23 \mu \mathrm{m}$. Inspection of Fig. 3 shows that these values agree qualitatively with the shifts of the left and right intensity peaks away from the adjacent boundaries, except for $\delta_{2}=23 \mu \mathrm{m}$. A $\delta_{2}$-value of approximately half of the length $l$ of the diffusion domain $(l=40 \mu \mathrm{m})$ indicates that the motional narrowing limit of a single peak has arisen, as is the case for Fig. $3(\mathrm{~d})$. A value $\delta_{2} \gg l$ would correspond to a single, narrowly focussed peak far into the motional narrowing limit.

One can introduce the unitless parameter $q=\delta_{2} / \frac{l}{2}$, i.e.,

$$
q=\sqrt[3]{\frac{8 \pi D}{\gamma G l^{3}}},
$$

as a control parameter for the extend of motional narrowing. $q$-values smaller than one, but not too small, indicate an enhancement of intensity near the edges of the dif- 
fusion domain, $q$-values larger than one indicate that the motional narrowing regime with a single intensity peak applies, and the magnitude of $q$ indicates how strongly peaked the intensity is.

The $q$-values for Fig. $3(\mathrm{a}-\mathrm{d})$ are listed in the caption. The values agree with the qualitative features in Fig. 3, i.e., the case $q<1$ corresponds to enhanced intensity near the boundary as in Figs. $3(\mathrm{a}, \mathrm{b}, \mathrm{c})$, the case $q>1$ corresponds to a single intensity peak as in Fig. $3(\mathrm{~d})$.

We now consider a spin ensemble that is homogeneously distributed in a spherical diffusion space which is outwardly bounded by an impermeable shell. In Fig. 5 we present, for spins diffusing within a sphere of radius $r_{a}=40 \mu \mathrm{m}$, frequency distortions given by the line shape function [15] and resulting from simulations. For stationary particles, a spherical diffusion domain will lead to a parabolic frequency spectrum reflecting the density distribution profile [16]: $r_{a}^{2}-z^{2}\left(|z| \leq r_{a}\right)$. The parabolic profile is easily recognized in Fig. 5 (a) as corresponding to slow diffusion $(D=$ $3 \cdot 10^{-7} \mathrm{~cm}^{2} / \mathrm{s}$ ) and is still apparent in Fig 5 (b) which corresponds to faster diffusion $\left(D=3 \cdot 10^{-6} \mathrm{~cm}^{2} / \mathrm{s}\right)$. Just as in the case of a one-dimensional geometry, diffusion leads to a decrease of the spectrum at the edges $\omega_{1} \approx-\gamma G r$ and $\omega_{n} \approx \gamma G r$ and induces two spectral peaks moving towards the center of the spectrum as the diffusion constant increases. The peaks in the three-dimensional case are not as clearly discernible as in the one-dimensional case (cf. Figs. 3 and 5). For slow diffusion small shoulders appear 
in the spectrum, only slightly distorting the parabolic density profile. Reconstruction of the spectra in Fig. 5 (a, b), however, will assign brightened, clearly visible shells of high spin density corresponding to the shoulders. Examples will be presented below. Figures $5(\mathrm{c}, \mathrm{d})$ for fast diffusion show more similarity to the corresponding one-dimensional cases, i.e., Figs. 3 (c,d). As the diffusion constant is increased the two shoulders merge (c) and finally form a single peak at the center (d).

As explained earlier, simulations have been conducted primarily to test the accuracy of the line shape function description. Such a test becomes especially germane in three dimensions where the theory has been advanced through various approximations. In comparing the results of the two methods as shown in Fig. 5, it can be seen that there are discrepancies, especially at the shoulders, but the main features agree well. The simulations have been carried out with spin ensembles of 160000 spins except for Fig. 5(d) for which case only 40000 spins were used. The large number of particles reduces statistical fluctuations and reveals the true shape of the spectra.

Since we use the same set of parameters in three dimensions that we applied in one dimension, the control parameter $q$ retains the values listed in the caption of Fig. 3 if we set $l=2 r$. Comparing the line shapes in Fig. 5 to those in Fig. 3, one can observe that the shoulders in Fig. 5 are farther away from the boundaries than the corresponding peaks in Fig. 3. This shift comes from the surrounding shell hindering free diffusion not only at the boundaries shown, but also to a certain degree 
at positions inside the diffusion interval - a consequence of a round rather than a cubic diffusion space. Nevertheless, $q$ can still be used to characterize the resulting line shape function.

More structure in the spectrum $I(\omega)$ emerges when an additional diffusion barrier (membrane) is placed into the system at radius $r_{i}<r_{a}$. Frequency spectra for such subdivided compartments are shown in Fig. 6. Inside each barrier the distortions to the spectrum are similar to those seen in Fig. 5. This can be noticed most easily in Fig. 6 (b) which clearly shows four frequency peaks, two stemming from the inner compartment, and two stemming from the outer compartment. It turns out that noticeable motional narrowing effects from a spherical boundary only occur inside that boundary. In Fig. 6 we see shoulders only on the inside of the boundaries. This is because only a small fraction of the spins in the outer compartment will be reflected by the inner membrane, and so the distortions on the outside are too small to be seen.

In Fig. 6 we can also consider the effect of the relative size of the diffusion domain. The parameter $q$ suggests that the size of the diffusion domain has an effect on $I(\omega)$ which is inverse to that of the diffusion constant. The values for $q$ (listed in figure caption) indicate that the motional narrowing effects caused by the inner shell are comparable to the one caused by the outer shell for a diffusion constant about ten times as high. The different influences on $I(\omega)$ of the size of the compartment and of the diffusion constant can also be seen when comparing Figs. 5 and 6 . It is of 
particular interest to note that Fig. 6 (c) shows a single intensity peak for the inner sphere, but two peaks for the outer shell, whereas Fig. 6 (d) shows a single peak both for the inner sphere and the outer shell. This behavior is in agreement with the qvalues listed in the figure caption: in case of Fig. 6 (c) $q=1.06$ for the inner sphere, $q=0.53$ for the outer shell, whereas in case of Fig. $6(\mathrm{~d})$ the $q$-values for both the inner sphere and the outer shell are larger than one.

Figures 5 and 6 demonstrate that the approximate line shape function description of the diffusion effects on MRI signals agrees well with the signals determined by simulations. The two methods agree least well in Fig. 6 (c) where the shoulders of the line shape function appear diminished in the simulation results. This is to be expected as a consequence of our approximation scheme. Nevertheless, a reconstruction of the simulated spectrum would produce essentially the same image as the spectrum derived from the line shape function: in both cases a bright ring will appear at a radius determined by the $q$-value for this figure. One can conclude that the line shape function description provides a tool with which we can proceed to investigate the influence of motional narrowing on the final product of microscopic MRI, a reconstructed image. 


\section{Reconstruction}

In this section we present two and three-dimensional reconstructions for the spherical diffusion domains investigated above. As input data we used the spectra calculated using the line shape function algorithm [15] to avoid artifacts caused by noise which would appear if simulated spectra were used. Since all the objects have spherical symmetry, a single frequency spectrum contains all the information necessary to reconstruct the three-dimensional structure of the corresponding original. Reconstruction methods are described in (27).

We use available reconstruction software (VIEWIT of NCSA at Urbana-Champaign) which employs a backprojection algorithm. The appearance of edge enhancement in reconstructed images requires frequency encoding in at least one direction since diffusion makes its mark solely on the frequency spectrum.

A two-dimensional reconstruction can be thought of as a projection of the frequency spectrum onto a plane (comparable to an X-ray image). Generally, one assumes the frequency spectrum to be proportional to the spin density. Figure 8 presents a set of such images for various objects and a range of diffusion constants.

The columns in Fig. 8 correspond to the four different geometries which are presented schematically in Fig. 7. In the outer columns we find the two structures investigated above: a spherical compartment in the left column and in the 
right column, a sphere of radius $r_{\text {inner }}$ surrounded by an outer shell with radius $r_{\text {outer }}$ where $r_{\text {inner }} / r_{\text {outer }}=2$. The two center columns portray impermeable hollow spheres (air bubbles) of radius $r_{\text {inner }}$ that are surrounded by filled shells that extend to $r_{\text {outer }}$. For the second column from the left $r_{\text {inner }} / r_{\text {outer }}=4$, and for the third one $r_{\text {inner }} / r_{\text {outer }}=2$. All objects have an outer diameter of $40 \mu \mathrm{m}$. The top row of Fig. 8 , representing densities undistorted by diffusion, gives an impression of the geometrical setting (cf. Fig. 7). The remaining rows represent reconstructed images for diffusion constants in the range $3 \cdot 10^{-7} \ldots 3 \cdot 10^{-4} \mathrm{~cm}^{2} / \mathrm{s}$.

The most obvious property of the images in Fig. 8 is that diffusion has a pronounced effect on the reconstructed images. Nearly all the images are different from each other, and, in comparing the images by row, one can see that the rate of diffusion determines the images as much as does the geometrical setting. At moderately low diffusion rates bright rings at the outside boundary appear as anticipated. As diffusion rates increase, these rings become brighter and move inwards until they finally form a single spot at the center of the spheres. The images in the bottom row show almost no difference between the different compartmental geometries, however, one needs to note that the diffusion constant assumed there $\left(3 \cdot 10^{-4} \mathrm{~cm}^{2} / \mathrm{s}\right)$ is ten times higher than that of pure water, and, therefore, such extreme motional narrowing will not occur in biological systems.

One can also see that motional narrowing effects can lead to misconceptions about 
the form of an object when image reconstruction techniques are applied without proper interpretation. Representative of a single cell, the spherical object imaged in the first column has been distorted by diffusion which has caused an edge to appear brighter and with a smaller diameter than the actual cell boundary. In the remaining three objects it is particularly interesting how the inner boundaries have been represented and in how far they resemble the actual objects. ${ }^{5}$ For such spheres as we consider here (i. e., with a radius on the order of $100 \mu \mathrm{m}$ or less) we see no evidence of any brightened rings surrounding the sphere. Images of hollow spheres exhibit instead a blurring of the inner boundaries rather than an edge enhancement. Yet, when the inner membrane serves also to confine water within, as in the column at the far right, a bright ring again appears, shrunken in diameter.

Motional narrowing effects are even more clearly pronounced in images reconstructed in three dimensions. As an example Fig. 9 presents such an image for the model depicted in Fig. 7 (d). The diffusion constant assumed is $3 \cdot 10^{-6} \mathrm{~cm}^{2} / \mathrm{s}$. Clearly visible in the image in Fig. 9 are the two shells of high intensity caused by the shoulders seen in Fig. 6 (b). Unlike the two-dimensional reconstruction in Fig. 8, the three-dimensional image shows the two shells at the same intensity. This implies that, in contrast to a two-dimensional reconstruction, the relative brightness of rings

\footnotetext{
${ }^{5}$ The hollow objects in column 2 and 3 could be, for example, air bubbles, but it should be pointed out that susceptibility differences may cause further distortions of such objects.
} 
and spots in a three-dimensional reconstruction does not provide information concerning the size of the actual object.

So far we have ignored relaxation and the corresponding broadening of the natural line width of a resonance signal. This broadening could, in principle, mask the diffusion effects shown, such as those in Fig. 9. In order to overcome this consequence of spin relaxation one needs a strong enough field gradient such that the natural line width is smaller than the features of the back-projected line shape function. To investigate the dependence on the natural line width, we carried out a corresponding line shape function calculation for a gradient ${ }^{6}$ of $0.02 \mathrm{~T} / \mathrm{m}$ and a $T_{2}$ value of $50 \mathrm{~ms}$, as can be found, e. g., in liver tissue (28). The geometrical setting and diffusion constant have been chosen as in Fig. 9. The reconstructed three-dimensional image is shown in Fig. 10. Because of the natural line width, the features in Fig. 10 are blurred to some extent compared to those in Fig. 9. Nonetheless, the image in Fig. 10 still shows the same structure as in Fig. 9; only the contrast is reduced. We conclude that for gradients of the order $0.02 \mathrm{~T} / \mathrm{m}$, relaxation does not eliminate the features that are a consequence of motional narrowing.

Another factor which can limit the appearance of motional narrowing effects in microscopic MRI of biological tissues is the permeability of biological boundaries. Prerequisite for motional narrowing effects are diffusion barriers which are largely

\footnotetext{
${ }^{6}$ Gradient fields of this strength are used in clinical MRI equipment.
} 
impermeable on the time scale of an MRI measurement. It is possible that not all biological boundaries are impermeable enough. Cell membranes, for example, often allow rapid passage of water. In addition, in cells the diffusion constant of water may decrease from the center towards the cell membrane which would make the motional narrowing effects discussed here less discernible.

To estimate the impermeability of a membrane, one can compare the average time $t_{1}$ it takes a particle inside a sphere of radius $r$ to diffuse to the boundary at $r$, $t_{1}=r^{2} / 6 D_{w}$, to the average time $t_{2}$ it takes a particle to leave the sphere. Assuming a membrane thickness $d$ and diffusion constant $D_{m}$ in the membrane, the latter time can be estimated to be $t_{2}=d R / 3 D_{m}(8)$. A list of permeabilities $P$ for liposome membranes of thickness $d$, as characterized by $P=D_{m} / d$, is provided in (29). It seems that the permeability of biological membranes, although often greater due to ion channels, is not too different from that of liposomes (30). If one considers the most permeable of liposome membranes studied in (29) characterized by $D_{m} / D_{w} \approx 10^{-4}$ and uses the geometry $r=10 \mu \mathrm{m}, d=5 \mathrm{~nm}$, one finds $t_{1} / t_{2} \approx 0.1$. One can conclude that about ten times as many particles reach the membrane and are reflected by it as pass through it. In other words, the majority of water molecules confined by a membrane with $D_{m} / D_{w}=10^{-4}$ will contribute to motional narrowing.

These results have encouraged us to suggest experiments which would specifically look for edge enhancement (31). Entirely within the realm of practicality would be 
images of ultra-thin water-filled glass capillaries, of only about $50 \mu \mathrm{m}$ in diameter. ${ }^{7}$ Since the achievable signal-to-noise ratio in an NMR experiment is proportional to the sample volume, special radio frequency coils are necessary for imaging samples less than $1 \mathrm{~mm}$ in diameter. A size reduction of the radio frequency coil improves coilsample coupling and increases the coil sensitivity. It has been shown previously (33) that pixel resolutions less than $10 \mu \mathrm{m}$ can be achieved using such coils. Initial measurements of spectroscopic linewidth, using samples of water within capillary tubes, indicate that a pixel resolution less than $3 \mu \mathrm{m}$ is attainable (32), which we predict would be sufficient to observe edge enhancement as long as the applied gradient is less than about $10 \mathrm{G} / \mathrm{cm}$.

\footnotetext{
${ }^{7}$ A specific design of such an experiment has been suggested by Peck, Webb, and Magin (32)
} 


\section{Conclusion}

We have demonstrated by means of computer simulations and a description in terms of Kubo's line shape function that diffusion plays an important role in generating image contrast on a microscopic length scale. Our investigation has led to the following interpretations of MRI micrographs: (i) intensity halos in an image point to the existence of otherwise invisible compartmental boundaries, (ii) water free volumes appear smaller than they actually are and may be overlaid by an intensity emanating from frequency distortions of the surrounding water, and (iii) motional narrowing effects within a cell can be regarded independently of the surrounding water.

We have discussed briefly two factors - natural line widths and permeable boundaries - that may offset or limit the appearance of motional narrowing effects in microscopic MRI of biological tissues. We have shown that both factors can certainly limit such effects, although neither should ordinarily be expected to eliminate motional narrowing effects.

Before motional narrowing effects are investigated in microscopic MRI of biological tissues, it should be fruitful to look for such effects in controllable phantom systems. In model systems such as a film of water between glass plates (1D case), water-filled glass capillaries (2D), or liposomes with a highly impermeable membrane (3D), edge enhancement should be observable. Such observations could demonstrate 
the viability of motional narrowing effects for edge enhancement in biological microscopic MRI. Experiments on a two-dimensional system (water-filled glass capillaries) are already under preparation (32).

\section{Acknowledgements}

We would like to thank Paul Lauterbur for useful discussions and suggestions. In numerous discussions Richard Magin gave us valuable insight into the experimental aspects involved in microscopic MRI. Lino Becerra helped us understand FFT techniques used in magnetic resonance experiments. We would also like to thank Robert Bittl for a program calculating line shape function functions and Clint Potter and Patrick Moran for their visualization programs VIEWIT and VT, respectively. The simulations have been computed on a Transputer-based parallel Computer at the Center for Parallel Computation for Molecular Dynamics at the University of Illinois supported by the National Institute of Health (grant 1 P41 RR05969*01). 


\section{Appendix:}

\section{A Analytic Solution for $m(z, t)$}

The solution for Eq. [3]

$$
\partial_{t} m_{\perp}=\left(D \partial_{z}^{2} \pm i \gamma G z\right) m_{\perp}
$$

can be determined by exploiting an analogy to the Schrödinger equation of a particle with charge $q$ in a homogeneous electric field $E$

$$
i \hbar \partial_{t} \Psi=\left(-\frac{\hbar^{2}}{2 \mu} \partial_{z}^{2}-q E z\right) \Psi
$$

Eq. [31] results from the following transformations to Eq. [32]: $\hbar \rightarrow 1, t \rightarrow i t$, $\frac{1}{2 \mu} \rightarrow D, q E \rightarrow i \gamma G$. The boundary condition [5], $m( \pm \infty, t)=0$, corresponds to the condition of vanishing wave function at infinity in the quantum mechanical case. The equivalence of [31] and [32] allows one to use the known Green's function of [32] $(34,35)$ for a spin ensemble with initial magnetization of Gaussian shape centered at $z_{o}$

$$
m(z, t=0)=\frac{1}{\sqrt{\pi \delta^{2}}} \exp \left(-\frac{(z-\bar{z})^{2}}{\delta^{2}}\right)
$$

yielding

$$
m(z, t)=\frac{1}{\sqrt{\pi \delta^{2}\left(1+\frac{4 D t}{\delta^{2}}\right)}} \exp \left[-\frac{\left((z-\bar{z}) \pm i D \gamma G t^{2}\right)^{2}}{\delta^{2}\left(1+\frac{4 D t}{\delta^{2}}\right)} \pm i \gamma G z t-\frac{\left(D \gamma^{2} G^{2} t^{3}\right)}{3}\right]
$$


as solution of [31].

The spatial integral of [34] $m(t)=\int_{-\infty}^{+\infty} d z m(z, t)$ describes the temporal behavior of a homogeneous overall magnetization of the spin ensemble. The integral reproduces the result of Hahn and Torrey $(14,13)$

$$
m(t) \propto e^{-\frac{D \gamma^{2} G^{2} t^{3}}{3}} .
$$

\section{B The Simulation Program}

The simulations assume that the observable magnetization results from a quantum mechanical ensemble average over a macroscopic number of nuclear magnetic spins and that the ensemble can be represented by a relatively small number of classical spin ensembles, each ensemble accounting for only a small fraction of the observable magnetization while representing a very large number of spins. [A similar description for electron spins was employed in (36).] Assuming this, we employ the Bloch equation for a classical description of the magnetization. Since microscopic interactions between different nuclear spins (e.g., dipole-dipole) are taken into account by the $T_{1}$ and $T_{2}$ parameters in the Bloch equations, the classical spins ensembles, hereafter simply called "spins," are non-interacting, and the total magnetization will be given by the vector sum over all the spins at any time during the simulation. We can thus illustrate the simulation algorithm by describing the steps performed for a single spin. 


\section{Step 1: Initialization}

At an initial position, chosen randomly within the accessible diffusion space, the magnetization is represented by a vector pointing in the negative $x$-direction to simulate an initial $\pi / 2$-pulse.

\section{Step 2: Relaxation}

Next, the Bloch equations are iterated according to Eq. [22]. If in the simulation one wishes to include magnetic field inhomogeneities in addition to the gradient field, it is necessary to switch to a coordinate system whose $z$-axis is aligned with the local magnetic field. This can be accomplished by applying the following transformation in the case that $B_{x}$ and $B_{y}$ are not both vanishing, i. e., the field is not already aligned with the $z$-axis,

$$
\vec{x} \rightarrow \boldsymbol{T}(\vec{B}) \vec{x}
$$

where the matrix $\boldsymbol{T}(\vec{B})$ is defined by

$$
\boldsymbol{T}(\vec{B})=\left(\begin{array}{ccc}
\frac{B_{x} B_{z}}{B B_{r}} & \frac{B_{y} B_{z}}{B B_{r}} & -\frac{B_{r}}{B} \\
-\frac{B_{y}}{B_{r}} & \frac{B_{x}}{B_{r}} & 0 \\
\frac{B_{x}}{B} & \frac{B_{y}}{B} & \frac{B_{z}}{B}
\end{array}\right)
$$

and where $B=|\vec{B}|$ and $B_{r}=\sqrt{B_{x}^{2}+B_{y}^{2}}$. In the study presented here no such field inhomogeneities were assumed, yet this feature can be quite useful in calculating magnetic relaxation under a variety of circumstances, including, for example, magnetic relaxation in the presence of superparamagnetic contrast agents. 
Transformation [37] is performed when appropriate, and then, for an aligned coordinate system the Bloch equations can be solved easily. For simplicity we consider only the deviation from equilibrium of the longitudinal magnetization by subtracting away the equilibrium magnetization,

$$
m_{z} \longrightarrow m_{z}-m_{z}^{o}
$$

With this last transformation the time evolution of the magnetization can be written as

$$
\vec{m}\left(t_{n+1}\right)=\boldsymbol{R}_{z}(\omega \Delta t) \boldsymbol{D} \vec{m}\left(t_{n}\right)
$$

where $\boldsymbol{D}$ and $\boldsymbol{R}_{z}(\omega \Delta t)$ denote the matrices describing decay and precession of $\vec{m}$, respectively. $\boldsymbol{D}$ and $\boldsymbol{R}_{z}(\omega \Delta t)$ are given by

$$
\boldsymbol{D}=\left(\begin{array}{ccc}
e^{-\Delta t / T_{2}} & 0 & 0 \\
0 & e^{-\Delta t / T_{2}} & 0 \\
0 & 0 & e^{-\Delta t / T_{1}}
\end{array}\right)
$$

and

$$
\boldsymbol{R}_{z}(\omega \Delta t)=\left(\begin{array}{ccc}
\cos \omega \Delta t & \sin \omega \Delta t & 0 \\
-\sin \omega \Delta t & \cos \omega \Delta t & 0 \\
0 & 0 & 1
\end{array}\right)
$$

The product $\boldsymbol{R}_{z}(\omega \Delta t) \boldsymbol{D}$ is then 


$$
\boldsymbol{R}_{z}(\omega \Delta t) \boldsymbol{D}=\left(\begin{array}{ccc}
e^{-\Delta t / T_{2}} \cos \omega \Delta t & \sin \omega \Delta t & 0 \\
-\sin \omega \Delta t & e^{-\Delta t / T_{2}} \cos \omega \Delta t & 0 \\
0 & 0 & e^{-\Delta t / T_{1}}
\end{array}\right)
$$

where $T_{1}$ and $T_{2}$ represent the times for longitudinal and transverse relaxation, $\omega$ the Larmor frequency for the local magnetic field and $\Delta t$ the length of a time step. We tried several time steps and used $\Delta t=50 \mathrm{~ms}$ in the simulations presented. After returning to laboratory coordinates by reversing transformation $[38]^{8}$ the new magnetization vector is obtained.

\section{Step 3: Diffusion}

Diffusion is simulated by Brownian motion of the spins. Each "diffusion step" is generated by a Monte-Carlo algorithm. The step width is taken from a distribution corresponding to that of Brownian motion at the same diffusion constant (see Eq. [21]). If the particle reaches a boundary it will be reflected. This reflection is performed so as to give a reasonable displacement by conserving the length of the step $(37,38)$.

Fig. 11 introduces the notation used to describe the reflection method. Take, for example, a spin which starts out at position $\vec{p}_{1}$ and moves towards $\vec{p}_{2}$, crossing a boundary at radius $R$. This step may be denoted as $\overrightarrow{\Delta p}=\vec{p}_{2}-\vec{p}_{1}$. The point $\vec{p}_{3}$

\footnotetext{
${ }^{8}$ Since $\boldsymbol{T}$ is a rotation matrix $\boldsymbol{T}^{-1}=\boldsymbol{T}^{T}$ holds.
} 
where the path crosses the boundary is given by

$$
\vec{p}_{3}=\vec{p}_{1}+\lambda \overrightarrow{\Delta p}
$$

with

$$
\lambda_{ \pm}=\frac{-\left(\overrightarrow{p_{1}} \cdot \overrightarrow{\Delta p}\right) \pm \sqrt{\left(\overrightarrow{p_{1}} \cdot \overrightarrow{\Delta p}\right)^{2}-|\overrightarrow{\Delta p}|^{2}\left(\left|\vec{p}_{1}\right|^{2}-R^{2}\right)}}{|\overrightarrow{\Delta p}|^{2}}
$$

The sign determines whether the reflection takes place inside or outside of the sphere.

For an inside reflection '+' has to be chosen, for an outside reflection '-', as shown in the right part of Fig. 11 . Knowing $\vec{p}_{3}$, the final position $\vec{p}_{2}{ }^{\prime}$ can be computed by

$$
\vec{p}_{2}^{\prime}=\vec{p}_{2}-2 \frac{\left(\vec{p}_{3}-\vec{o}\right) \cdot\left(\vec{p}_{2}-\vec{p}_{3}\right)}{R^{2}}\left(\vec{p}_{3}-\vec{o}\right)
$$

where $\vec{o}$ represents the center of the sphere.

\section{Step 4: Data acquisition}

After applying steps 2 and 3 a pre-defined number of times, an observable like the spin magnetization can be extracted. This may be either the total magnetization or, e.g., just the $x$-component, or even derived information like the frequency distribution of the magnetization.

Steps 2 through 4 are repeated until the desired simulation time has elapsed. 


\section{References}

[1] C. B. Ahn and Z. H.Cho, Med. Phys., 16, 22 (1988).

[2] J. B. Aguayo, S. J. Blackband, J. Schoeniger, M. A. Mattingly, and M. Hintermann, Nature, 322, 190 (1986).

[3] Z. H.Cho, Med. Phys., 15, 815 (1989), and references therein.

[4] R. Kubo: "Stochastic Theory of Line Shape", in D. ter Haar, editor, "Fluctuation, Relaxation and Resonance in Magnetic Systems", 23. Oliver and Boyd, Edinburgh, 1962.

[5] P. T. Callaghan: "Principles of NMR Microscopy". Oxford University Press, 1991.

[6] B. Pütz: "Diffusionseffekte in der Kernspintomographie", Thesis, Physics Department, Technical University of Munich, May 1990.

[7] R. Brasch, H.-J. Weinmann, and G. Wesbey, AJR, 142, 625 (1984).

[8] D. Barsky, B. Pütz, K. Schulten, and R. L. Magin, Magnetic Resonance in Medicine (in press), (1990).

[9] G. Kabalka, E. Buonocore, K. Hubner, T. Moss, N. Norley, and L. Huang, Radiology, 163, 255 (1987). 
[10] E. C. Unger, T. Winokur, P. MacDougall, J. Rosenblum, M. Clair, R. Gatenby, and C. Tilcock, Radiology, 171, 81 (1989).

[11] H.-J. Weinmann, R. Brasch, W.-R. Press, and G. Wesbey, AJR, 142, 619 (1984).

[12] C. P. Slichter: "Principles of Magnetic Resonance". Springer Verlag, 1990.

[13] H. C. Torrey, Phys. Rev., 104, 563 (1956).

[14] E. L. Hahn, Phys. Rev., 80, 580 (1950).

[15] R. Kubo, Jour. Math. Phys., 4(2), 174 (1963).

[16] W. H. Press, B. P. Flannery, S. A. Teukolsky, and W. T. Vetterling: "Numerical Recipes in C". Cambridge University Press, 1988.

[17] R. Bittl and K. Schulten, J. Chem. Phys, 90(3), 1794 (1989).

[18] J. Crank: "The Mathematics of Diffusion". Oxford Science Press, 1975.

[19] G. E. Forsythe and W. R. Wasow: "Finite-Difference Methods for Partial Differential Equations", in Applied Mathematics Series. John Wiley \& Sons, Inc., New York London, 1960.

[20] W. Nadler and K. Schulten, J. Chem. Phys., 82, 151 (1985).

[21] W. Nadler and K. Schulten, J. Chem. Phys., 84, 4015 (1986). 
[22] R. Bittl: "Stochastische Quantenmechanik magnetfeldabhängiger Reaktionen von Dublettpaaren", PhD Thesis, Physics Department, Technical University of Munich, 1988.

[23] W. Nadler, P. Tavan, and K. Schulten, Europ. Biophys. J., 12, 25 (1985).

[24] H.-U. Bauer, K. Schulten, and W. Nadler, Phys. Rev. B, 38, 445 (1988).

[25] R. R. Ernst, G. Bodenhausen, and A. Wokaun: "Principles of Nuclear Magnetic Resonance in One and Two Dimensions". Clarendon Press, Oxford, 1987.

[26] T. C. Farrar and E. D. Becker: "Pulse and Fourier Transform NMR. Introduction to Theory and Methods". Academic Press, 1971.

[27] A. Rosenfeld and A. C. Kak: "Digital Picture Processing", 353, Academic Press, 1982.

[28] D. D. Stark, W. G. Bradley, et al.: "Magnetic Resonance Imaging". The C. V. Mosby Company, St. Louis, 1988.

[29] R. L. Magin, M. R. Niesman, and G. Bacic: "The influence of fluidity on membrane permeability: Correspondence between studies of membrane models and simple biological systems", in R. C. Aloia, C. C. Curtain, and L. M. Gorden, editors, "Advances in Membrane Fluidity, vol. 4". A. R. Liss, New York, in press.

[30] R. B. Gennis: "Biomembranes". Springer Verlag, 1989. 
[31] B. Pütz, D. Barsky, and K. Schulten, Chem. Phys. Letters (in Press), (1991).

[32] T. Peck, A. Webb, and R. L. Magin: "private communication".

[33] T. Peck, R. Magin, and P. Lauterbur, Abstracts, 9th Annual Meeting SMRM, $270270(1990)$.

[34] W. Franz: "Quantentheorie". Springer, 1970.

[35] C. G. Darwin, Proc. Roy. Soc. A, 154, 61 (1936).

[36] K. Schulten and I. R. Epstein, J. Chem. Phys., 71, 309 (1979).

[37] G. Lamm and K. Schulten, J. Chem. Phys., 75, 365 (1981).

[38] G. Lamm and K. Schulten, J. Chem. Phys., 78, 2713 (1983). 


\section{List of Figures}

1 Relation between measured frequency spectrum and spin distribution for a spin ensemble under the influence of a linear gradient field. The density distribution along the $z$-axis is projected onto a spectral function (spectrum) by the gradient. This spectral function is the observable, and the spin distribution is then deduced from it. Schematically shown are two spectra which could be observed from the transverse magnetization of a homogeneous spin distribution. The rectangular $(-)$ spectrum would result from fixed spins while for diffusing spins the peaked (- -) spectrum would be observed as a result of motional narrowing. When the spins remain fixed, the rectangular frequency spectrum reflected about the field gradient yields the actual spin density. In case of sufficiently fast diffusion, the frequency spectrum, when reflected about the field gradient, yields an artificially focussed spin concentration. 
2 Contributions $I\left(\omega, z_{k}\right)$ to the line shape function for one-dimensional diffusion, calculated from $I\left(\omega, z_{k}\right)=\operatorname{Re}\left(c_{k}(\omega)\right)$. The $z$-axis represents the diffusion interval $\left[-z_{b}, z_{b}\right]$ whose length is $40 \mu \mathrm{m}$. The frequency interval shown $\left[\gamma\left(H_{o}-G z_{1}\right), \gamma\left(H_{o}+G z_{n}\right)\right]$ corresponds to the frequency range one would observe without diffusion. ( $D=10^{-5} \mathrm{~cm}^{2} / \mathrm{s}, G=$ $0.02 \mathrm{~T} / \mathrm{m}$.

$3 \quad$ Frequency spectra $I(\omega)$ for one-dimensional diffusion in a $40 \mu \mathrm{m}$ interval. The smooth curves correspond to the line shape function evaluated according to Eq. [15]. The jagged curves have been determined by simulation, according to Eq. [23] and as described in the section, A Simulation Program. The bounds of the diffusion domain are indicated by vertical dashed lines. The frequency is shown as $\omega / \gamma G$ in $\mu \mathrm{m}$. The field gradient is $0.02 \mathrm{~T} / \mathrm{m}$. The diffusion constants assumed are: a) $3 \cdot 10^{-7}$, b) $3 \cdot 10^{-6}$, c) $3 \cdot 10^{-5}$, and d) $3 \cdot 10^{-4} \mathrm{~cm}^{2} / \mathrm{s}$. The q-values (introduced later) are: a) 0.11, b) 0.24, c) 0.53, and d) 1.14. Simulated were 160000 particles. 
4 Schematic explanation of motional narrowing near compartmental walls. Each figure (a, b, c) shows representative spins diffusing in a one-dimensional diffusion space as time evolves from top to bottom. The spin paths are shown as solid lines, their average positions over the presented paths are shown by vertical dashed lines. The rate of diffusion increases from (a) to (c): (a) $D \ll(\Delta z)^{2} / \Delta T$, (b) $D \approx$ $(\Delta z)^{2} / \Delta T,(\mathrm{c}) D \gg(\Delta z)^{2} / \Delta T$.

5 Comparison of spectra for diffusion in a sphere of diameter $40 \mu \mathrm{m}$. (smooth curves: line shape function jagged curves: simulation results) The parameters chosen are the same as in case of Fig. 3. The corresponding $q$-values [30] are the same as those listed in the caption of Fig. 3. Simulated were 160000 particles for (a-c), 40000 for (d).

6 Comparison of spectra for diffusion in a subdivided sphere. Inside a sphere of radius $20 \mu \mathrm{m}$ an impermeable sphere of $10 \mu \mathrm{m}$ radius is centered, both spheres are indicated by the dashed lines. Smooth: line shape function, jagged: simulation, parameters as in Fig. 3. The corresponding $q$-values [30] are as follows: for the $20 \mu \mathrm{m}$ radius a) 0.11 , b) 0.24 , c) 0.53 , and d) 1.14 ; the corresponding values are double for the $10 \mu \mathrm{m}$ radius. Simulated were 200000 particles for (a-c), 40000 for (d). 
$7 \quad$ A pictorial guide of the models whose images appear in Fig. 8. The order of the columns in Fig. 8 is the same as the horizontal order here. The image reconstructions have been performed for four spherical models; each $20 \mu \mathrm{m}$ in radius, and (a) containing uniform spin density, (b) a hollow center $5 \mu \mathrm{m}$ in radius separated by a membrane from the surrounding uniform spin density, (c) a spherical membrane $10 \mu \mathrm{m}$ in radius otherwise as (b), and (d) containing a uniform spin density that is divided by a membrane at a radius of $10 \mu \mathrm{m}$. The boundaries shown are impermeable, the shading indicates the spin distribution.

8 Two-dimensional reconstructions from frequency spectra. Geometries are described in Fig. 7. The width of each square is $40 \mu \mathrm{m}$. Diffusion constants assumed are from top to bottom: no diffusion (undistorted image), $3 \cdot 10^{-7}, 3 \cdot 10^{-6}, 3 \cdot 10^{-5}, 3 \cdot 10^{-4} \mathrm{~cm}^{2} / \mathrm{s}$. The intensities cannot be compared directly because the visualization program automatically scales the images so that they use the whole grey scale. In the image at the far right of row 4 , the center has been cut out to make the outer ring visible. 
9 Three-dimensional reconstruction for the case of a spherical compartment of radius $r_{\text {inner }}$ surrounded by spherical shell with $r_{\text {inner }}=\frac{1}{2} r_{\text {outer }}$ as in Fig. 7 (right). Diffusion constant assumed is $3 \cdot 10^{-6} \mathrm{~cm}^{2} / \mathrm{s}$. The surrounding cube has sides of length $40 \mu \mathrm{m}$. The $q$-values are 0.24 and 0.49 for the outer and inner shell, respectively.

10 The effect of lifetime broadening on the three-dimensional reconstruction of the same object as shown in Fig. 9. A $T_{2}$ value of $50 \mathrm{~ms}$ and a field gradient of $0.02 \mathrm{~T} / \mathrm{m}$ was assumed.

11 Left: Definition of points used to describe reflections. Right: Correct choice of the sign of $\lambda$ in [41]. 


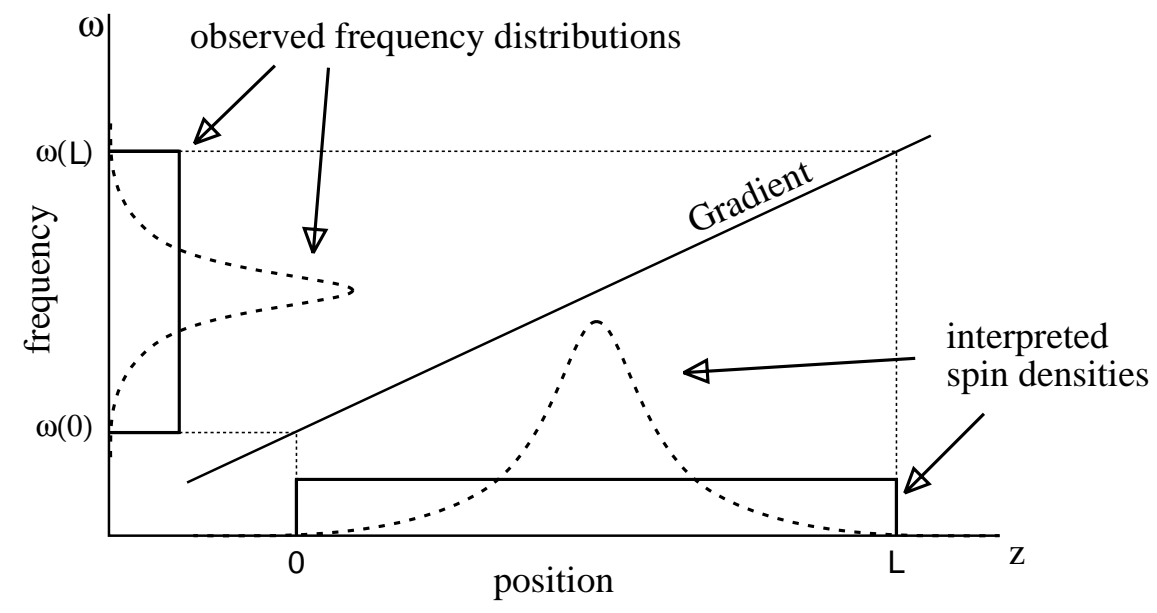

Figure 1: Relation between measured frequency spectrum and spin distribution for a spin ensemble under the influence of a linear gradient field. The density distribution along the $z$-axis is projected onto a spectral function (spectrum) by the gradient. This spectral function is the observable, and the spin distribution is then deduced from it. Schematically shown are two spectra which could be observed from the transverse magnetization of a homogeneous spin distribution. The rectangular (-) spectrum would result from fixed spins while for diffusing spins the peaked (- -) spectrum would be observed as a result of motional narrowing. When the spins remain fixed, the rectangular frequency spectrum reflected about the field gradient yields the actual spin density. In case of sufficiently fast diffusion, the frequency spectrum, when reflected about the field gradient, yields an artificially focussed spin concentration. 


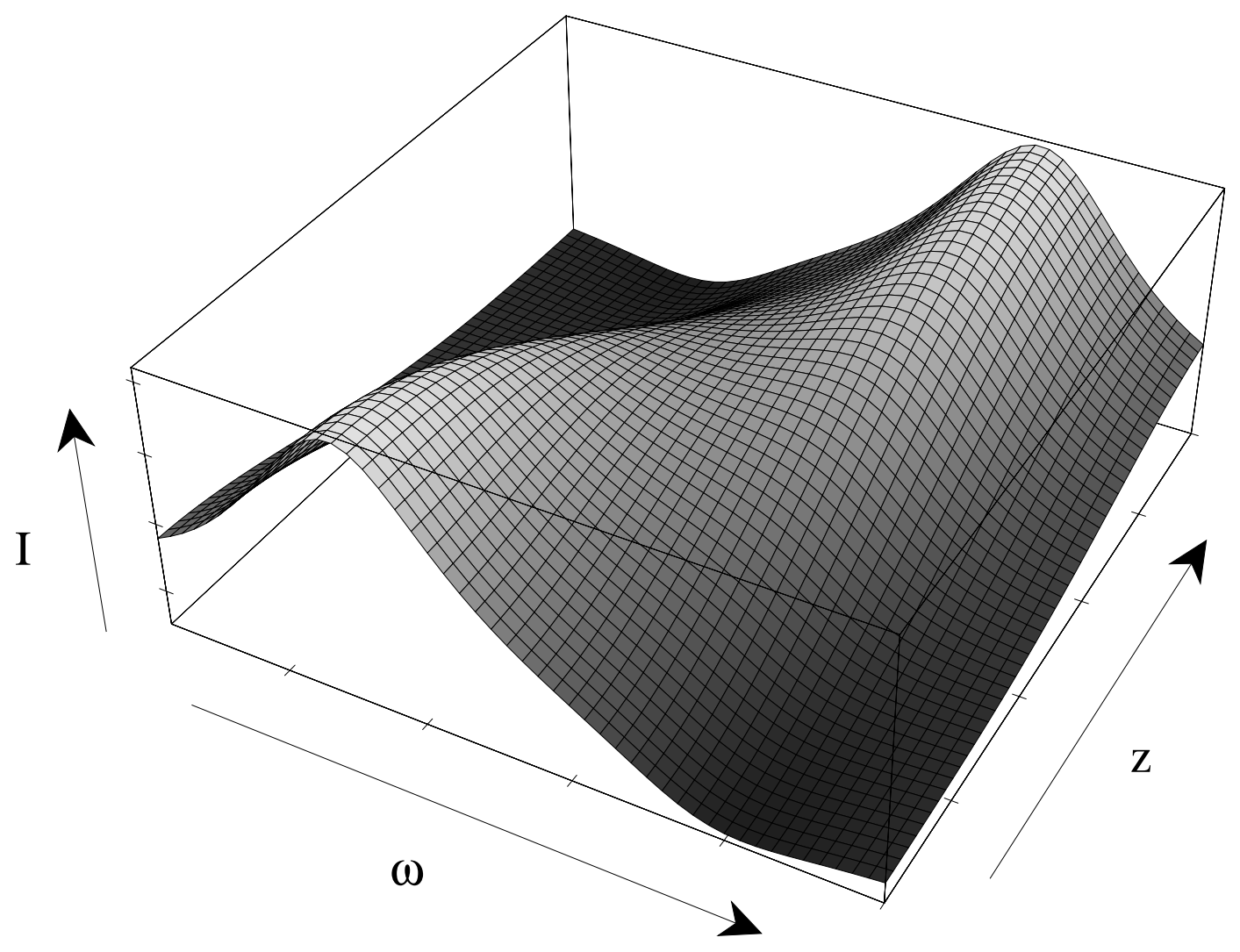

Figure 2: Contributions $I\left(\omega, z_{k}\right)$ to the line shape function for one-dimensional diffusion, calculated from $I\left(\omega, z_{k}\right)=\operatorname{Re}\left(c_{k}(\omega)\right)$. The $z$-axis represents the diffusion interval $\left[-z_{b}, z_{b}\right]$ whose length is $40 \mu \mathrm{m}$. The frequency interval shown $\left[\gamma\left(H_{o}-G z_{1}\right), \gamma\left(H_{o}+G z_{n}\right)\right]$ corresponds to the frequency range one would observe without diffusion. ( $D=10^{-5} \mathrm{~cm}^{2} / \mathrm{s}, G=0.02 \mathrm{~T} / \mathrm{m}$.) 

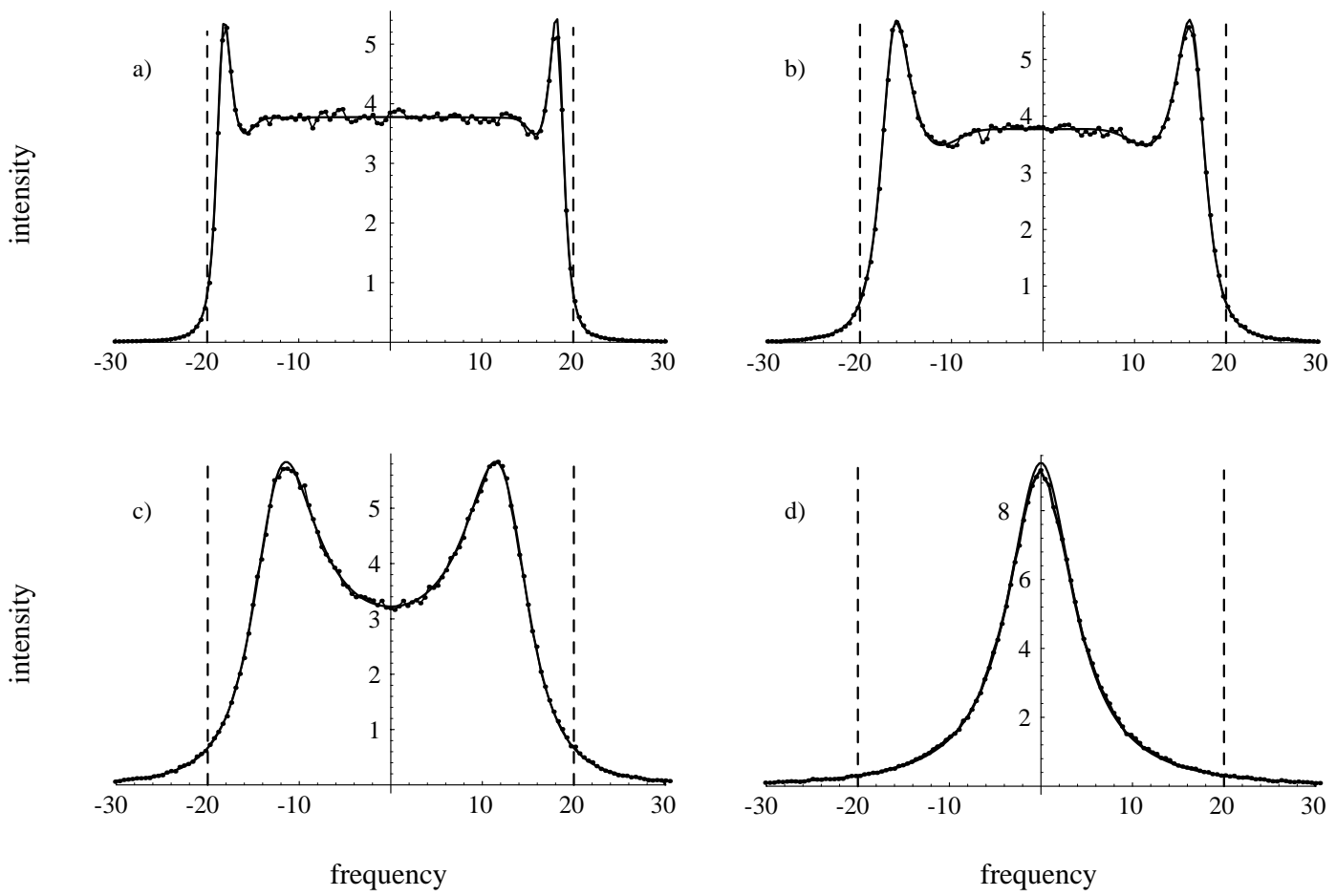

Figure 3: Frequency spectra $I(\omega)$ for one-dimensional diffusion in a $40 \mu \mathrm{m}$ interval. The smooth curves correspond to the line shape function evaluated according to Eq. [15]. The jagged curves have been determined by simulation, according to Eq. [23] and as described in the section, A Simulation Program. The bounds of the diffusion domain are indicated by vertical dashed lines. The frequency is shown as $\omega / \gamma G$ in $\mu \mathrm{m}$. The field gradient is $0.02 \mathrm{~T} / \mathrm{m}$. The diffusion constants assumed are: a) $3 \cdot 10^{-7}$, b) $3 \cdot 10^{-6}$, c) $3 \cdot 10^{-5}$, and d) $3 \cdot 10^{-4} \mathrm{~cm}^{2} / \mathrm{s}$. The q-values (introduced later) are: a) 0.11, b) 0.24, c) 0.53 , and d) 1.14. Simulated were 160000 particles. 
a)

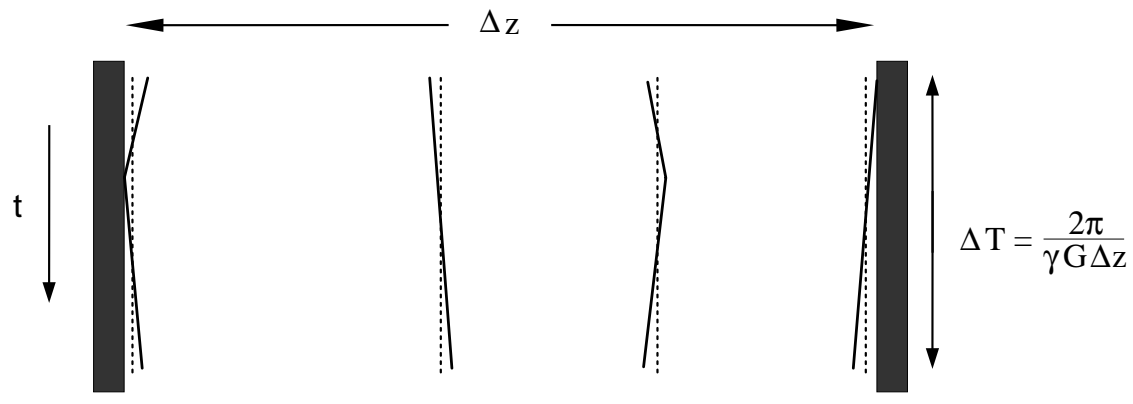

b)

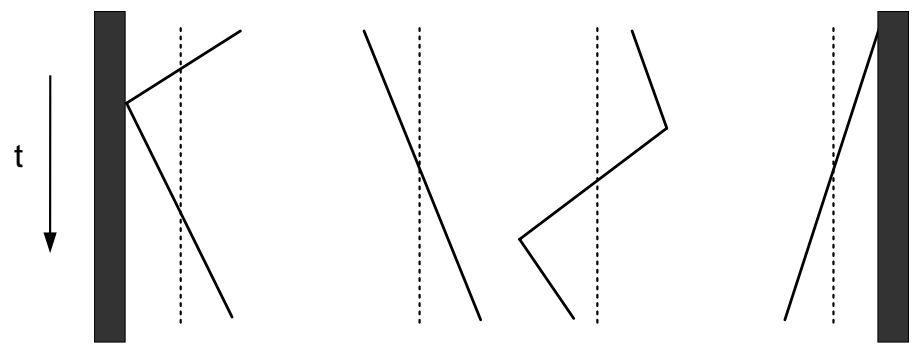

c)

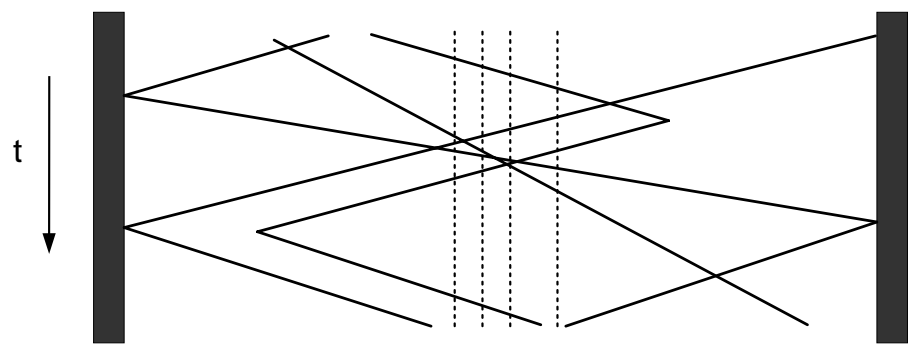

Figure 4: $\quad$ Schematic explanation of motional narrowing near compartmental walls. Each figure $(\mathrm{a}, \mathrm{b}, \mathrm{c})$ shows representative spins diffusing in a one-dimensional diffusion space as time evolves from top to bottom. The spin paths are shown as solid lines, their average positions over the presented paths are shown by vertical dashed lines. The rate of diffusion increases from (a) to (c): (a) $D \ll(\Delta z)^{2} / \Delta T$, (b) $D \approx(\Delta z)^{2} / \Delta T$, (c) $D \gg(\Delta z)^{2} / \Delta T$. 

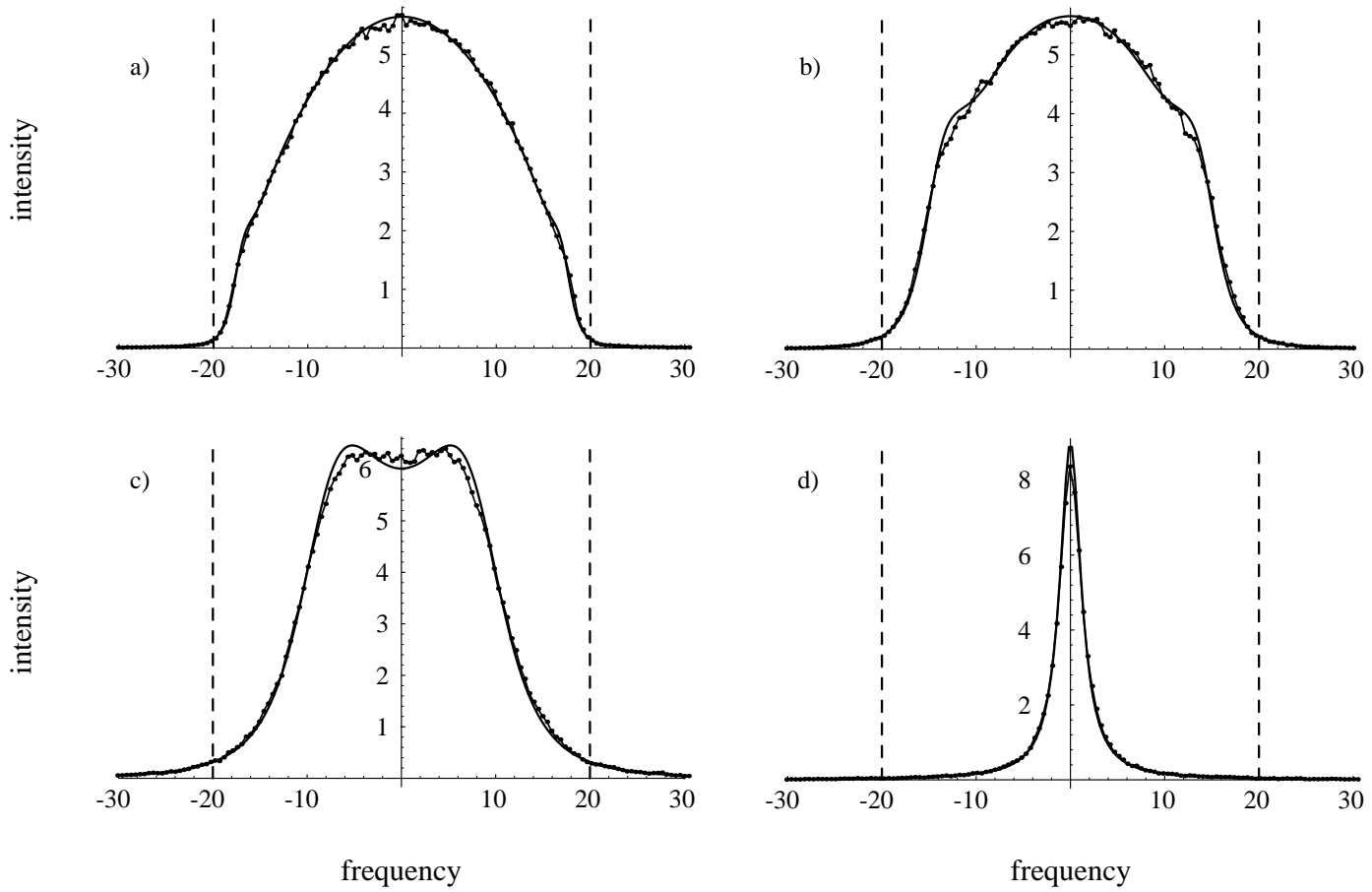

Figure 5: Comparison of spectra for diffusion in a sphere of diameter $40 \mu \mathrm{m}$. (smooth curves: line shape function jagged curves: simulation results) The parameters chosen are the same as in case of Fig. 3. The corresponding $q$-values [30] are the same as those listed in the caption of Fig. 3. Simulated were 160000 particles for (a-c), 40000 for (d). 

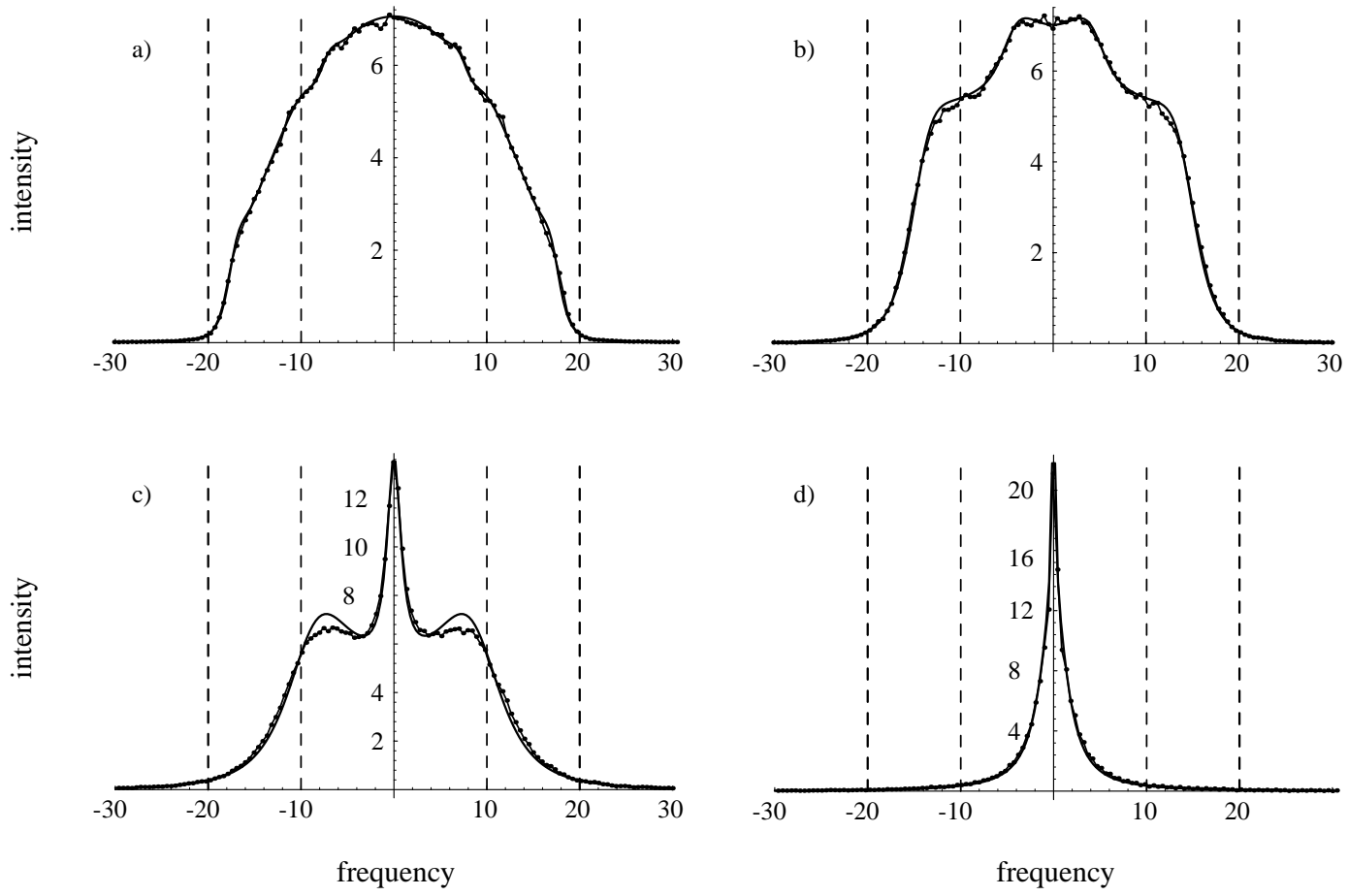

Figure 6: Comparison of spectra for diffusion in a subdivided sphere. Inside a sphere of radius $20 \mu \mathrm{m}$ an impermeable sphere of $10 \mu \mathrm{m}$ radius is centered, both spheres are indicated by the dashed lines. Smooth: line shape function, jagged: simulation, parameters as in Fig. 3. The corresponding $q$-values [30] are as follows: for the $20 \mu \mathrm{m}$ radius a) 0.11, b) 0.24 , c) 0.53 , and d) 1.14 ; the corresponding values are double for the $10 \mu \mathrm{m}$ radius. Simulated were 200000 particles for (a-c), 40000 for (d). 


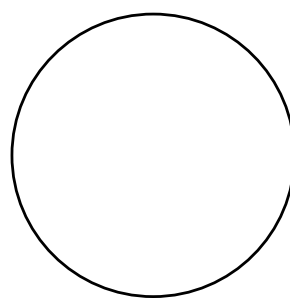

a

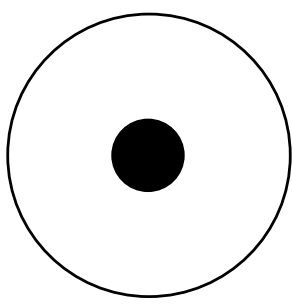

b

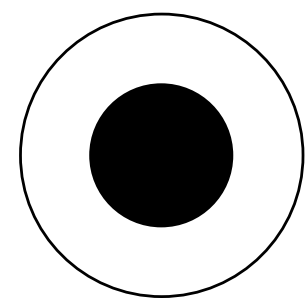

c

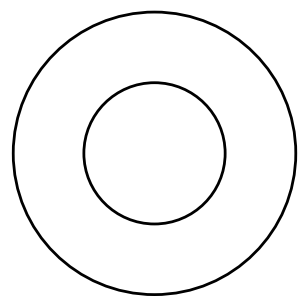

d

Figure 7: A pictorial guide of the models whose images appear in Fig. 8. The order of the columns in Fig. 8 is the same as the horizontal order here. The image reconstructions have been performed for four spherical models; each $20 \mu \mathrm{m}$ in radius, and (a) containing uniform spin density, (b) a hollow center $5 \mu \mathrm{m}$ in radius separated by a membrane from the surrounding uniform spin density, (c) a spherical membrane $10 \mu \mathrm{m}$ in radius otherwise as (b), and (d) containing a uniform spin density that is divided by a membrane at a radius of $10 \mu \mathrm{m}$. The boundaries shown are impermeable, the shading indicates the spin distribution. 

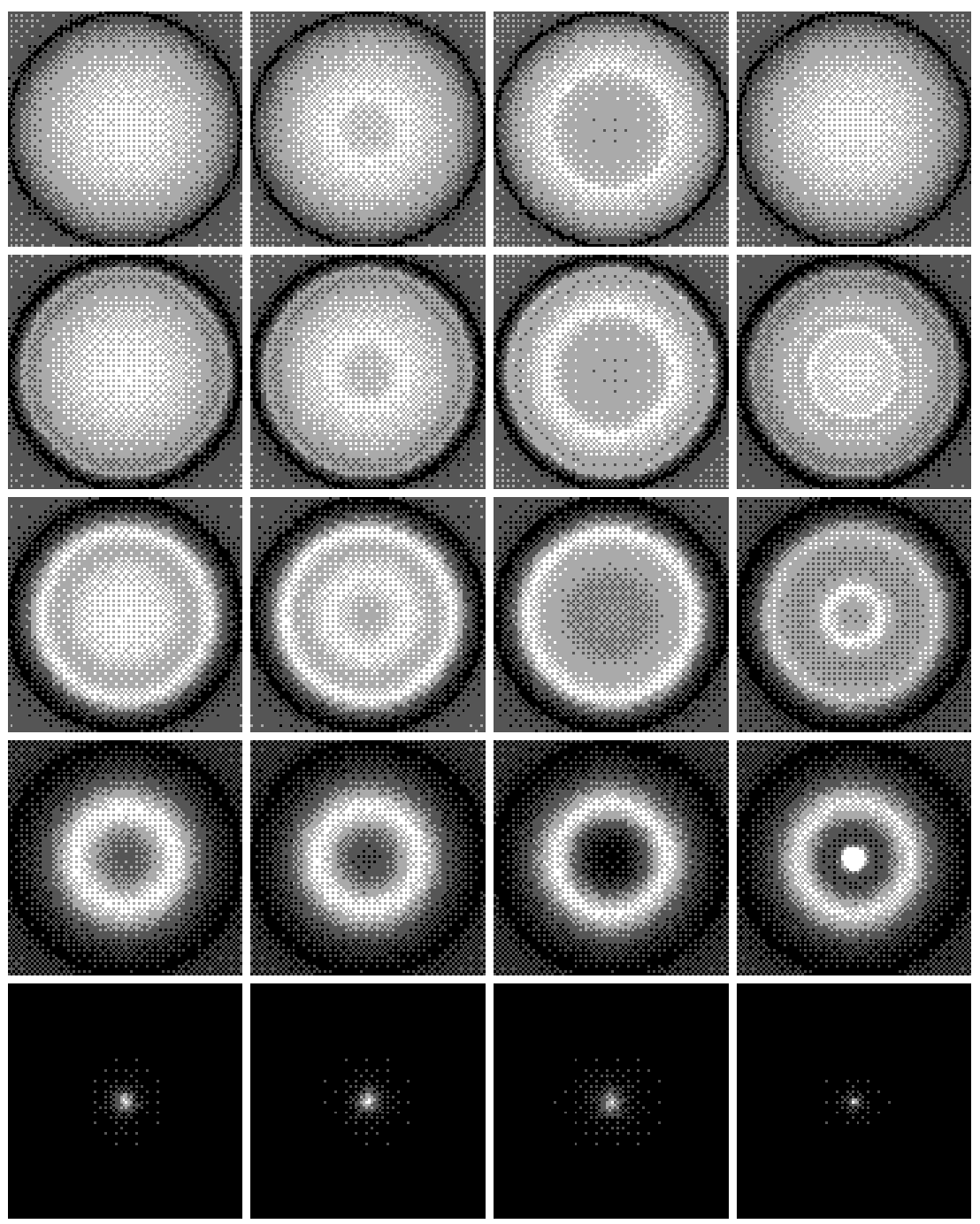

Figure 8: Two-dimensional reconstructions from frequency spectra. Geometries are described in Fig. 7. The width of each square is $40 \mu \mathrm{m}$. Diffusion constants assumed are from top to bottom: no diffusion (undistorted image), $3 \cdot 10^{-7}, 3 \cdot 10^{-6}$, $3 \cdot 10^{-5}, 3 \cdot 10^{-4} \mathrm{~cm}^{2} / \mathrm{s}$. The intensities cannot be compared directly because the visualization program automatically scales the images so that they use the whole grey scale. In the image at the far right of row 4 , the center has been cut out to make the outer ring visible. 


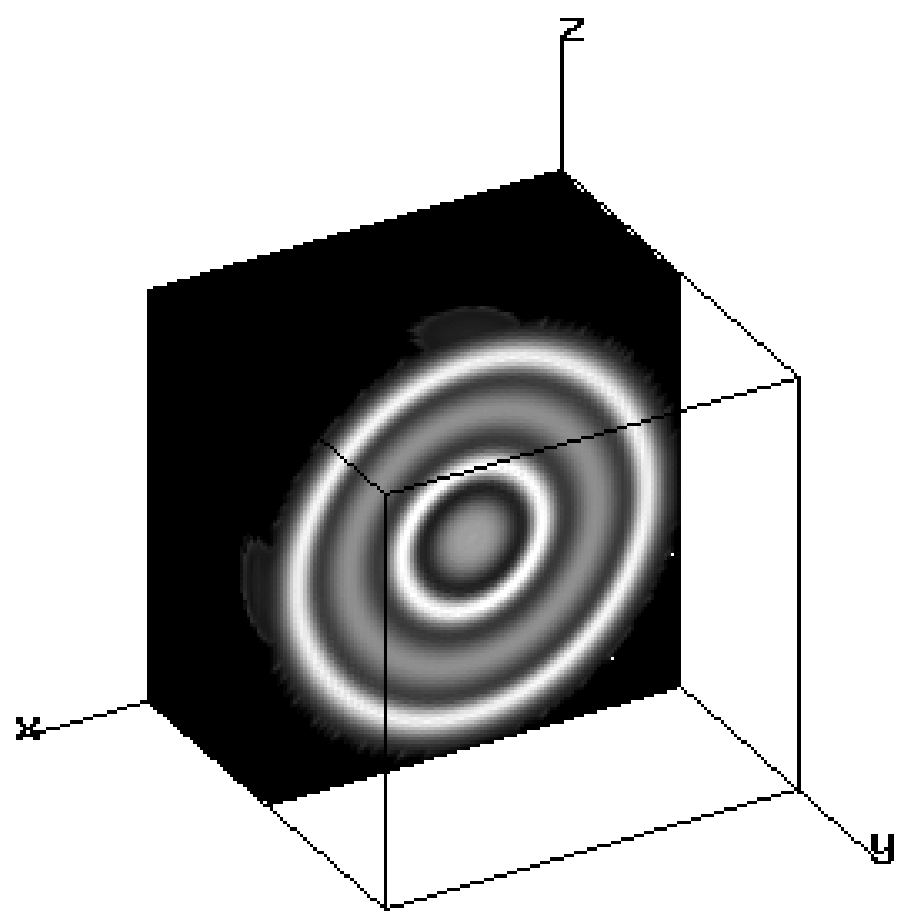

Figure 9: Three-dimensional reconstruction for the case of a spherical compartment of radius $r_{\text {inner }}$ surrounded by spherical shell with $r_{\text {inner }}=\frac{1}{2} r_{\text {outer }}$ as in Fig. 7 (right). Diffusion constant assumed is $3 \cdot 10^{-6} \mathrm{~cm}^{2} / \mathrm{s}$. The surrounding cube has sides of length $40 \mu \mathrm{m}$. The $q$-values are 0.24 and 0.49 for the outer and inner shell, respectively. 


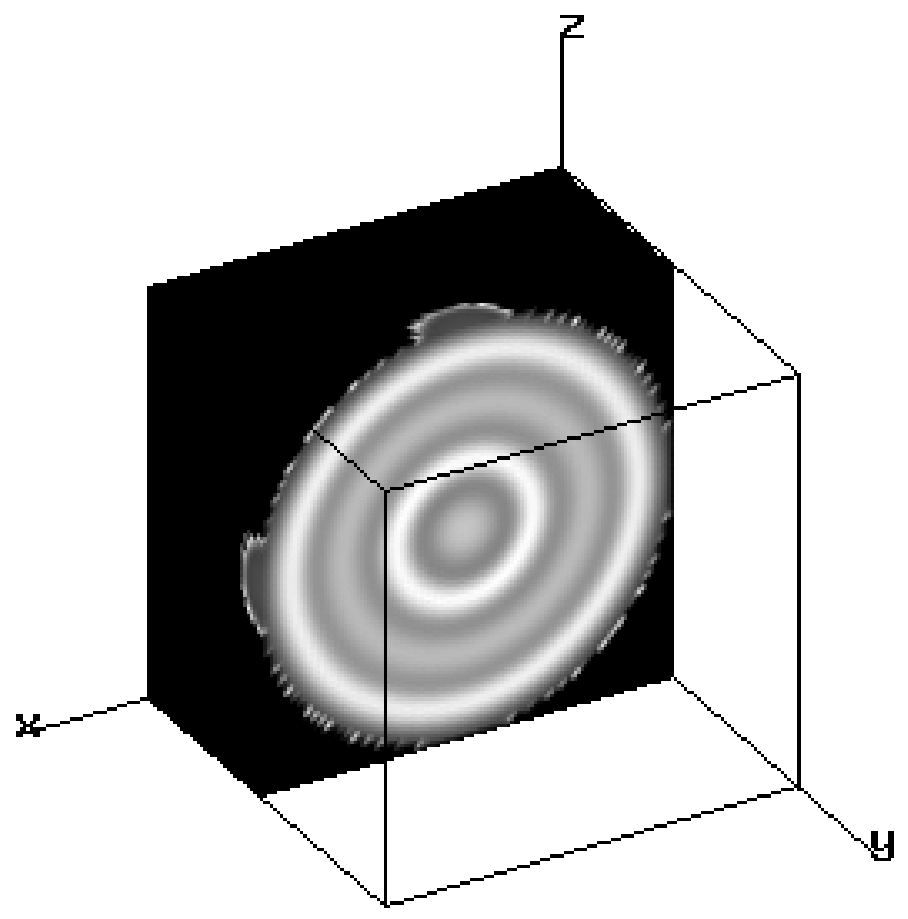

Figure 10: The effect of lifetime broadening on the three-dimensional reconstruction of the same object as shown in Fig. 9. A $T_{2}$ value of $50 \mathrm{~ms}$ and a field gradient of $0.02 \mathrm{~T} / \mathrm{m}$ was assumed. 

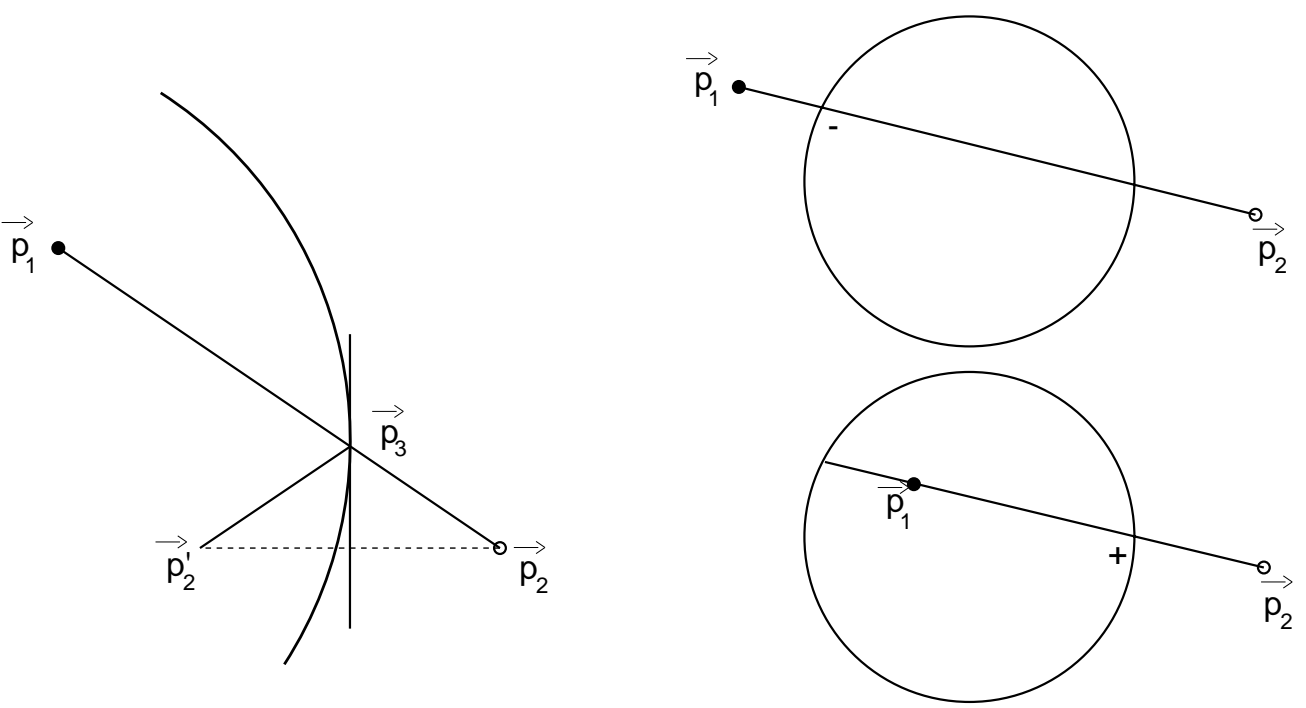

Figure 11: Left: Definition of points used to describe reflections. Right: Correct choice of the sign of $\lambda$ in [41]. 